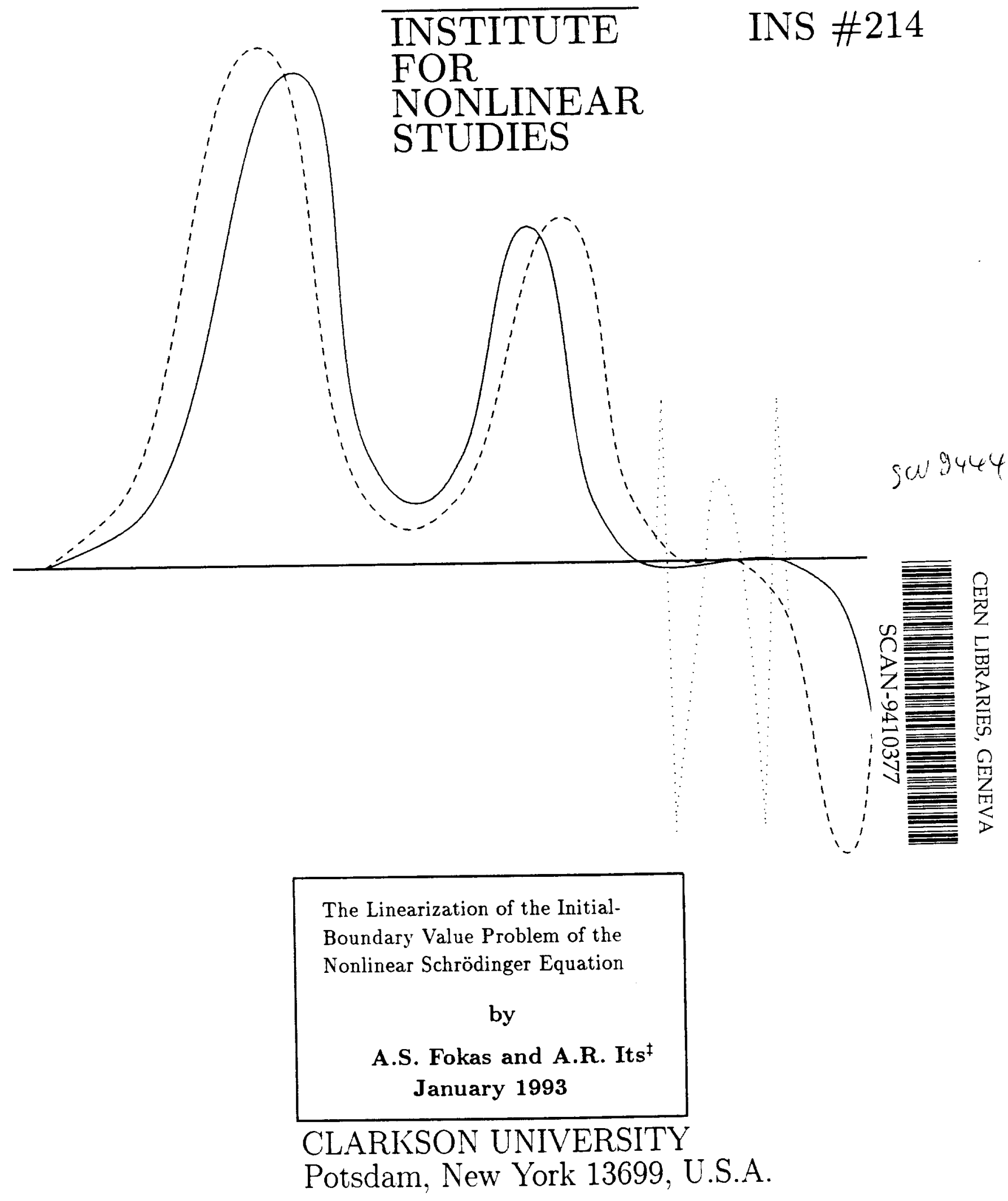

Ion leave of absence from St. Petersburg University, St. Petersburg, Russia 


\title{
The Linearization of the Initial-Boundary Value Problem of the Nonlinear Schrödinger Equation
}

\author{
A.S. Fokas and A.R. Its ${ }^{\dagger}$, \\ Department of Mathematics and Computer Science \\ and Institute for Nonlinear Studies \\ Clarkson University \\ Potsdam, New York 13699-5815, U.S.A.
}

January 1993

\section{INS \#214}

\begin{abstract}
We consider the nonlinear Schrödinger equation with both $x$ and $t$ in $[0, \infty)$. We assume that $q(x, 0)=u(x)$ and $q(0, t)=v(t)$ are given, that $u(0)=v(0)$, and that $u(x), v(t)$, as well as their first two derivatives belong to $L_{1} \cap L_{2}\left(\mathbb{R}^{+}\right)$. We show that the solution of this initial-boundary value problem can be reduced to solving a Riemann-Hilbert ( $\mathrm{RH})$ problem in the complex $k$-plane with jumps on $\operatorname{Im}\left(k^{2}\right)=0$. This $\mathrm{RH}$ problem is equivalent to a linear integral equation which has a unique global solution. This linear integral equation is uniquely defined in terms of certain functions (scattering data) $b(k)$ and $c(k)$. The function $b(k)$ can be effectively computed in terms of $u(x)$. However, although the analytic properties of $c(k)$ are completely determined, the relationship between $c(k), u(x)$ and $v(t)$ is highly nonlinear. In spite of this difficulty we can give an effective description of the asymptotic behavior of $q(x, t)$ for large $t$. In particular we show that as $t \rightarrow \infty$, solitons are generated moving away from the boundary. In addition, our formalism can be used to generate effectively pairs of functions $q(0, t)$ and $q_{x}(0, t)$ compatible with a given $q(x, 0)$ as well as to determine the associated $q(x, t)$. It is important to emphasize that the analysis of this problem, in addition to techniques of exact integrability, requires the essential use of general PDE techniques.
\end{abstract}

${ }^{\dagger}$ On leave of absence from St. Petersburg University, St. Petersburg, Russia. 


\section{Introduction}

For integrable equations, a method exists for solving the initial-value problem on the infinite line for decaying initial data. For evolution equations in one spatial variable, this method reduces the solution of the Cauchy problem to the formulation of a certain classical mathematical problem called the Riemann-Hilbert $(\mathrm{RH})$ problem. A RH problem can be solved via a linear integral equation. A distinguished property of integrable equations is that they can be written as the compatibility condition of a pair of linear eigenvalue equations, called the Lax pair [1]. The associated RH problem is essentially determined by the $x$-part of the Lax pair; the $t$-part of the Lax pair plays only an auxiliary role. In the case of the nonlinear Schrödinger (NLS) equation, the relevant RH problem is formulated in the complex $k$-plane with a jump on $\operatorname{Im}(k)=0$.

Many physical problems are formulated as initial-boundary value problems. For example, such a problem arises in the modeling of certain ionospheric experiments, when one directs a radio frequency wave at the ionosphere. At the reflection point of the wave, a sufficient level of electron plasma waves is excited and nonlinearity becomes important. This problem gives rise to the NLS equation with $x, t \epsilon[0, \infty)[2]$. Furthermore, several other physical problems can be reduced to initialboundary value problems. For example, such a problem arises in connection with optical switches [3], and can also be modeled by a NLS. The occurrence of the Korteweg-de Vries equation on the quarter plane is discussed in [20]-[24].

Recently a new formalism has been developed [4], for studying initial-boundary value problems on the half-infinite line for decaying initial and boundary data. This formalism also reduces the solution of the initial-boundary value problem to the solution of a single RH problem. However, for the formulation of this $\mathrm{RH}$ problem, both the $x$ - and the $t$-parts of the Lax pair play an important role. Actually, it is the $t$-part which determines where, in the complex $k$-plane, the jumps occur. In the case of the NLS the jumps occur on $\operatorname{Im}\left(k^{2}\right)=0$, which is a reflection of the fact that the $t$-part of the Lax pair contains $k^{2}$, which in turn is a consequence of the fact that the NLS involves a second derivative in $x$. Here we study the initial-boundary value problem for the NLS equation in detail. We show that the analysis of this problem, in addition to techniques from exact integrability, it also requires the essential use of more general PDE techniques. In the cases studied so far, the exact methods could be used to establish existence of global solutions as well as to study the properties of these solutions. In contrast, in the problem studied here exact methods are used only to study the properties of solutions. It shows that a hybrid between exact methods and general PDE techniques, can provide a powerful approach for analyzing problems of mathemaitcal and physical significance. 
We expect that a wide class of problems can be analyzed in a similar manner.

We consider the NLS equation

$$
i q_{t}+q_{x x}-2 \lambda|q|^{2} q=0, \quad x, t \epsilon[0, \infty) ; \quad \lambda= \pm 1
$$

where $q(x, 0)=u(x)$ and $q(0, t)=v(t)$ are given. We assume that

$$
\begin{gathered}
u(x) \epsilon H_{2}\left(\mathbb{R}^{+}\right), v(t) \epsilon C_{2}\left(\mathbb{R}^{+}\right), u(0)=v(0), x u(x) \text { and } x^{2} u(x) \epsilon L_{2}\left(\mathbb{R}^{+}\right), v(t) \epsilon L_{1} \cap L_{2}\left(\mathbb{R}^{+}\right), \\
v^{\prime}(t), \quad t v(t), \quad t v^{\prime}(t), \quad t v^{\prime \prime}(t) \epsilon L_{1}\left(\mathbb{R}^{+}\right),
\end{gathered}
$$

where $H_{2}$ denotes that a function and its first two derivatives belong to $L_{2}, C_{2}$ denotes that a function is twice differentiable, and prime denotes differentiation.

The cases $\lambda=1$ and $\lambda=-1$ are usually referred to as the defocusing and focusing cases, respectively. Equation (1.1) is the compatibility condition of the following Lax pair for the $2 \times 2$ matrix $w(x, t, k)[5]$,

$$
\begin{gathered}
w_{x}+i k \sigma_{3} w=Q w \\
w_{t}+U w=w C(t), \quad U(x, t, k) \doteqdot 2 i k^{2} \sigma_{3}+i \lambda|q|^{2} \sigma_{3}-2 k Q+i Q_{x} \sigma_{3},
\end{gathered}
$$

where $\sigma_{3}=\operatorname{diag}(1,-1)$, the $2 \times 2$ matrix $C(t)$ is an arbitrary function of $t$, and $Q(x, t)$ is an off-diagonal matrix with 12 and 21 entries given by $q$ and $\lambda \bar{q}$, respectively.

We have developed the following linearization scheme for the solution of the initial-boundary value problem of the NLS. Given $q(x, 0)$ construct $s_{1}^{+}$and $s_{2}^{+}$by $s_{1}^{+}(k) \doteqdot \psi_{1}(0, k), s_{2}^{+}(k) \doteqdot \psi_{2}(0, k)$, where $\left(\psi_{1}(x, k), \psi_{2}(x, k)\right)^{T}$ is the solution of $(1.3 \mathrm{a})$ with $q(x, t)$ replaced by $q(x, 0)$, satisfying the boundary condition $\lim _{x \rightarrow \infty}\left[\left(\psi_{1}, \psi_{2}\right)^{T} \exp (-i k x)\right]=(0,1)^{T}$. Define $b(k)$ by $b=s_{1}^{+} / \overline{s_{2}^{+}}$. Let $c(k)$, $k \in \mathbb{R}^{-} \cup i \mathbb{R}^{+}$be the boundary value of a function meromorphic for $k \epsilon I I$ (I, II, III, IV denote the first, second, third, and fourth quadrants of the complex $k$-plane), with poles at the zeros of $s_{2}^{+}(k)$ and at the points $\left\{k_{j}\right\}_{1}^{N}, k_{j} \in I I$ which are assumed to be different than the zeros of $s_{2}^{+}(k)$ (generic case); let $c_{j}$ denote the residues of $c(k)$ at $k_{j}$; also $c(k) \rightarrow 0$ as $k \rightarrow \infty$. Having $s_{1}^{+}(k), s_{2}^{+}(k)$ and $c(k)$ solve a RH problem for a $2 \times 2$ meromorphic function $\hat{Z}_{p}$ with possible poles only at $\left\{k_{j}\right\}_{1}^{N}$. This $\mathrm{RH}$ problem is depicted in Figure 1.1. Finally determine $q(x, t)$ by $q(x, t)=2 i \lim _{k \rightarrow \infty}\left(k \hat{Z}_{p}(x, t, k)\right)_{12}$, 
have the meaning of the discrete spectrum of equation (1.3b) evaluated at $x=0$ and supplemented with the boundary condition $w_{1} s_{2}^{+}(k)-w_{2} s_{1}^{+}(k)=0$ at $t=0$ ) give rise to solitons which always
move away from the boundary.

$$
\left(\begin{array}{cc}
1 & 0 \\
c(k) e^{\theta} & 1
\end{array}\right)
$$

$$
\begin{array}{r}
\left(\begin{array}{cc}
1 & -\lambda \bar{c} e^{-\theta} \\
0 & 1
\end{array}\right)\left(\begin{array}{cc}
1 & -b e^{-\theta} \\
\lambda \bar{b} e^{\theta} & 1-\lambda|b|^{2}
\end{array}\right)^{-1}\left(\begin{array}{cc}
1 & 0 \\
c e^{\theta} & 1
\end{array}\right) \frac{I I}{I I I} \mid \begin{array}{cc}
I \\
I V
\end{array} \\
\text { Figure 1.1 } \\
\left(\begin{array}{cc}
1 & -\lambda \overline{c(\bar{k})} e^{-\theta} \\
0 & 1
\end{array}\right)
\end{array}
$$

\section{The RH problem associated with the initial-boundary value problem of NLS.}

The $x, t$ dependence enters only through $\theta(x, t)=2 i\left(k x+2 k^{2} t\right)$.

In the case of $q(x, 0)=0$ the $\mathrm{RH}$ problem reduces to the one depicted in Figure 1.2; it is specified by the boundary values of $c(k)$ on $k \in \mathbb{R}^{-} \cup i \mathbb{R}^{+}$, by $\left\{k_{j}\right\}_{1}^{N}$, and by $\left\{c_{j}\right\}_{1}^{N}$.

$$
\begin{aligned}
& \left(\begin{array}{cc}
1 & 0 \\
c(k) e^{\theta} & 1
\end{array}\right) \\
& \left(\begin{array}{cc}
1-\lambda|c(k)|^{2} & -\lambda \overline{c(k)} e^{-\theta} \\
c(k) e^{\theta} & 1
\end{array}\right) \frac{I I}{I I I+} \mid \begin{array}{cc}
\mid \\
I V
\end{array}\left(\begin{array}{cc}
1 & 0 \\
0 & 1
\end{array}\right) \\
& \left(\begin{array}{cc}
1 & -\lambda \overline{c(\bar{k})} e^{-\theta} \\
0 & 1
\end{array}\right)
\end{aligned}
$$

Figure 1.2

The RH problem for the focusing NLS with of $q(x, 0)=0$. The $x, t$ dependence enters only through $\theta(x, t)=2 i\left(k x+2 k^{2} t\right)$. 
Unfortunately, although we have a complete characterization of the analytic properties of $c(k)$, we have not found an effective way of computing $c(k)$ in terms of $q(0, t)$ and $q(x, 0)$. (For more details see the discussion below). In spite of this fact we can give an effective description of the long time behavior $(\lambda=-1)$ :

$$
q(x, t)=-2 \eta_{j} \frac{\exp \left[-2 i \xi_{j} x-4 i\left(\xi_{j}^{2}-\eta_{j}^{2}\right) t-i \varphi_{j}\right]}{\cosh \left[2 \eta_{j}\left(x+4 \xi_{j} t\right)-\Delta_{j}\right]}+0\left(t^{-\frac{1}{2}}\right), t \rightarrow \infty,-\frac{x}{4 t}=\xi_{j}+0\left(\frac{1}{t}\right), j=1, \ldots, N
$$

where

$$
\begin{gathered}
\eta_{j}=\operatorname{Im}\left(k_{j}\right), \quad \xi_{j}=\operatorname{Re}\left(k_{j}\right) \\
\varphi_{j}=-\frac{\pi}{2}+\arg c_{j}+\sum_{l=1}^{N}\left[\operatorname{sign}\left(\xi_{l}-\xi_{j}\right)-1\right] \arg \left(\frac{k_{j}-k_{l}}{k_{j}-\bar{k}_{l}}\right)+\frac{1}{\pi} \int_{-\infty}^{-x / 4 t} \frac{\log \left[1+|b(\mu)+\overline{c(\mu)}|^{2}\right]}{\left(\mu-\xi_{j}\right)^{2}+\eta_{j}^{2}}\left(\mu-\xi_{j}\right) d \mu \\
\Delta_{j}=-\log 2 \eta_{j}+\log \left|c_{j}\right|+\sum_{l=1}^{N}\left[\operatorname{sign}\left(\xi_{l}-\xi_{j}\right)-1\right] \log \left|\frac{k_{j}-k_{l}}{k_{j}-\bar{k}_{l}}\right|-\frac{\eta_{j}}{\pi} \int_{-\infty}^{-x / 4 t} \frac{\log \left[1+|b(\mu)+\overline{c(\mu)}|^{2}\right]}{\left(\mu-\xi_{j}\right)^{2}+\eta_{j}^{2}} d \mu .
\end{gathered}
$$

All $k_{j} \epsilon I I$, thus all $\xi_{j}<0$ and the solitons move away from the boundary. The summation terms in the above equations describe the interaction among solitons, while the integration terms describe the interaction between solitons and the dispersive part.

Discussion The linearization scheme developed in this paper can be summarized as follows: Given $q(x, 0)$, construct $b(k)$. Then, if $c(k)$ is any suitably decaying function meromorphic for $k \epsilon I I$, the solution of the $\mathrm{RH}$ problem of Figure 1.1 generates the solution $q(x, t)$ corresponding to initial data $q(x, 0)$ and some boundary data $q(0, t)$. The main limitation of our result is that for given $q(x, 0)$ and $q(0, t)$ we cannot construct $c(k)$ by solving a linear problem. Nevertheless, we claim that for any given $q(x, 0)$ and $q(0, t)$ satisfying $(1.2)$, the corresponding function $c(k)$ exists; in other words, the $\mathrm{RH}$ problem of Figure 1.1 solves the initial-boundary value problem (1.1) for general initial-boundary data.

The above $\mathrm{RH}$ problem is quite natural. To appreciate this we first recall the $\mathrm{RH}$ problem that corresponds to the NLS with $x \epsilon(-\infty, \infty)$. This $\mathrm{RH}$ problem is depicted in Figure 1.3. 


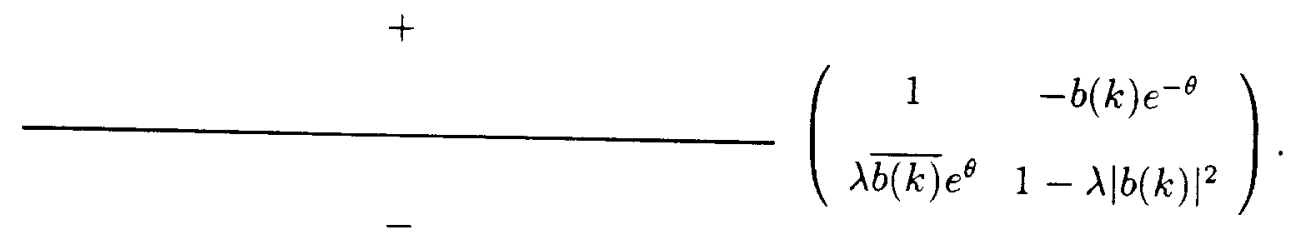

Figure 1.3

Comparing the RH problems of Figure 1.1 and Figure 1.3 we see that the jumps for $k \in \mathbb{R}^{+}$are identical. The jump for $k \epsilon i \mathbb{R}^{+}$can not have a nonzero entry in the 12 position since $e^{-\theta}$ is unbounded for $k \epsilon i \mathbb{R}^{+}$. The jump for $k \epsilon i \mathbb{R}^{-}$follows by symmetry considerations. Finally the jump for $k \in \mathbb{R}^{-}$ follows from the cyclic condition that the product of the jump matrices equals unity (this is a reflection of continuity at $k=0$ ). The fact that $c(k)$ has analytic continuation for $k \epsilon I I$ can also be easily understood. At $t=0$, the RH problem of Figure 1.1 must be reduced to the one that defines $q(x, 0)$. At $t=0$, the term $e^{\theta}$ has analytic continuation in $I I$. Thus the jumps along the imaginary axis can be mapped to a jump on the negative real axis. In this way, at $t=0$ one finds the RH problem of Figure 1.3 with $\theta$ replaced by $2 i k x$. This RH problem corresponds precisely to $q(x, 0)$.

The fact that $q(x, 0), q(0, t)$ and $c(k)$ are related in a nonlinear way is a reflection of the fact that $q_{x}(0, t)$ depends nonlinearly on $q(x, 0)$ and $q(0, t)$. To appreciate this we first recall the solution of the linearized problem

$$
i q_{t}+q_{x x}=0, \quad x, t \epsilon[0, \infty),
$$

where $q(0, t)$ and $q(x, 0)$ are given and decaying for large $t$ and large $x$. This problem can be solved by the sine transform. However, in order to draw comparisons with the nonlinear problem we shall use a Fourier transform

$$
\hat{q}(k, t)=\int_{0}^{\infty} d x e^{i k x} q(x, t) .
$$

The evolution of the Fourier data $\hat{q}(k, t)$ is given by

$$
\hat{q}_{t}+i k^{2} \hat{q}=i q_{x}(0, t)+k q(0, t) .
$$

In equation $(1.8), q(0, t)$ is known but $q_{x}(0, t)$ is unknown (the sine transform is precisely used in order to eliminate $\left.q_{x}(0, t)\right)$. This apparently ominus situation can be bypassed by using the fact 
that the solution $\hat{q}(k, t)$ of $(1.8)$ is analytic in the upper half of the $k$ complex plane. It turns out that this requirement implies

$$
\hat{q}(k, 0)=-\int_{0}^{\infty} d t e^{i k^{2} t}\left(i q_{x}(0, t)+k q(0, t)\right)
$$

Given $q(x, 0)$ and $q(0, t)$, and using the substitution $k=e^{i \pi / 4} \sqrt{\rho}, \rho>0$, equation (1.9) yields $q_{x}(0, t)$. It is important to notice that if $q_{x}(0, t)$ and $q(0, t)$ are arbitrary functions, then the rhs of equation (1.9) will be analytic for $k \epsilon I \cup I I I$. However, in order for $q_{x}(0, t)$ and $q(0, t)$ to be the boundary values of the solution of equation (1.6), it is necessary and sufficient that the rhs of equation (1.9) has analytic continuation across the positive imaginary $k$ axis $(\hat{q}(k, 0)$ is analytic for $k \in I \cup I I)$.

Before discussing the nonlinear problem, we emphasize that using these type of analyticity arguments, it is possible to solve linear equations for which the standard spectral theory fails. An example of such an equation is the linearized $\mathrm{KdV} u_{t}+u_{x x x}=0$, for which there does not exist a proper generalization of the sine transform.

We now discuss the nonlinear problem. Let $\left(\hat{\psi}_{1}(t, k), \hat{\psi}_{2}(t, k)\right)^{T}$ be the solution of the vector equation

$$
\hat{\psi}_{t}+\left(2 i k^{2} \sigma_{3}+i \lambda|q(0, t)|^{2} \sigma_{3}-2 k Q(0, t)+i Q_{x}(0, t) \sigma_{3}\right) \hat{\psi}=0,
$$

satisfying the boundary condition $\lim _{t \rightarrow \infty}\left[\left(\hat{\psi}_{1}, \hat{\psi}_{2}\right) \exp \left(-2 i k^{2} t\right)=(0,1)^{T}\right.$. Let $r(k) \doteqdot \hat{\psi}_{1}(0, k) / \hat{\psi}_{2}(0, k)$. It turns out that for arbitrary decaying functions $q(0, t)$ and $q_{x}(0, t), r(k)$ is a meromorphic function for $k \epsilon I \cup I I I$ and $r(k) \rightarrow 0$ as $k \rightarrow \infty$. However, if $q(0, t)$ and $q_{x}(0, t)$ are the boundary values of the NLS then in addition $r(k)$ satisfies

$$
r(k)=\frac{s_{1}^{+}(k)}{s_{2}^{+}(k)}, k \epsilon I
$$

where $s_{1}^{+}$and $s_{2}^{+}$are determined from $q(x, 0)$ and are analytic for $k \epsilon I \cup I I$. Equation (1.11) is the analogue of equation (1.9). It shows that although the relationship between $q(x, 0), q(0, t)$, and $q_{x}(0, t)$ is highly nonlinear, its reflection in the $k$-plane (scattering space) is rather simple: $r(k)$ has analytic continuation across the positive imaginary $k$ axis.

There exists an invertible correspondence between the "potential" $\left\{q(0, t), q_{x}(0, t)\right\}$ and the scattering data $r(k)$ : Given $q(0, t)$ and $q_{x}(0, t)$, equation (1.10) implies $r(k)$. Conversely given a meromorphic function $r(k)$, one can find $q(0, t)$ and $q_{x}(0, t)$ by solving a RH problem with the jump $r(k)$ for $k^{2} \in \mathbb{R}$. This provides an effective way for deriving pairs of functions $q(0, t)$ and $q_{x}(0, t)$ 
compatible with a given $q(x, 0)$. Indeed, given $q(x, 0)$ one first computes $r(k)$ for $k \epsilon I$ from equation
(1.11). Let $r(k), k \in I I I$ be any (1.11). Let $r(k), k \in I I I$ be any suitably decaying meromorphic function. solution of the above $\mathrm{RH}$
problem yields $q(0, t)$ and $q_{x}(0, t)$.

Unfortunately, given $q(0, t)$ and $q(x, 0)$ we cannot compute $r(k)$ for $k \epsilon I I I$ by solving a linear problem. This is a consequence of the fact that now we have a "mixed" problem where one gives "half" the potential, i.e. $q(0, t)$ and "half" the scattering data, i.e. $r(k)$, for $k \in I$. It turns out that this problem can be formulated as a nonlinear $\mathrm{RH}$ problem and will be discussed elsewhere.

The study of the large $t$ behavior of $q(x, t)$ reduces to the study of the large $t$ behavior of the $\mathrm{RH}$ problem of Figure 1.1. Because the $x, t$ dependence of this $\mathrm{RH}$ problem is rather simple, it is possible to give an effective asymptotic description of $q(x, t)$ as $t \rightarrow \infty$.

It was mentioned earlier that the analysis of equation (1.1) requires an essential use of general PDE techniques. This follows from the fact that in order to study the map between $\left\{q(0, t), q_{x}(0, t)\right\}$ and $r(k)$ one needs apriori estimates for $q_{x}(0, t)$. The uniqueness and existence of a global solution for the NLS on the quarter plane is established in [12]. This result makes fundamental use of certain equations which are the analogues of the first three conserved quantities. Therefore, this theory uses $L_{2}$ estimates. However, the methods of exact integrability are based on $L_{1}$ estimates. It is difficulties. In this paper, following ideas of [13] we show how to get $L_{1}$ estimates for $q(x, t)$ for all $t \geq 0$. The more difficult problem of obtaining $L_{1}$ estimates for $q_{x}(0, t)$ is discussed in [13].

There exist certain particular boundary conditions for which the difficulties discussed above disappear and the problem can be solved by exact methods. Such cases are discussed in [14]-[19]. data that appear in the boundary problem for the elliptic version of data the boundary problem for the version of sine-Gordon equation [25].

Notation We will use the following notation: overbar denotes complex conjugation; $a^{+}(k)$ denotes a function analytic for $k \in \mathbb{C}^{+}$(upper half of the complex $k$-plane); $a^{-}(k)$ denotes a function analytic for $k \epsilon \mathbb{C}^{-} ; \hat{a}^{+}(k)$ denotes a function analytic for $k \epsilon I$ (first quadrant) UIII (third quadrant); $\hat{a}^{-}(k)$ denotes a function analytic for $k \epsilon I I$ (second quadrant) $\cup I V$ (fourth quadrant); the subscript $p$ denotes meromorphicity instead of analyticity, for example $\hat{a}_{p}^{+}(k)$ is meromorphic for $k \epsilon I \cup I I I$; if the matrix $A$ is denoted by $A=\left(A^{+}, A^{-}\right)$, then $A^{+}=\left(A_{1}^{+}, A_{2}^{+}\right)^{T}$ and $A^{-}=\left(A_{1}^{-}, A_{2}^{-}\right)^{T}$, denote the
first and the second columns on the matrix $A ; I \doteqdot \operatorname{diag}(1,1)$. 
Summary of Results In $\S 2$ we present the relevant formalism. (a) In 2.1 we analyze the $x$ part of the Lax pair, i.e. equation (1.3a). We define the $2 \times 2$ matrices $\varphi(x, t, k)$ and $\psi(x, t, k)$ as the solutions of equation (1.3a) specified by the boundary conditions $\varphi(0, t, k)=I$, and $\lim _{x \rightarrow \infty}$ $\psi(x, t, k) \exp \left(i k x \sigma_{3}\right)=I$, respectively. We define the matrices $\Phi(x, t, k)$ and $\Psi(x, t, k)$ by the equations $\Phi(x, t, k)=\varphi(x, t, k) \exp \left(i k x \sigma_{3}\right)$ and $\Psi(x, t, k)=\psi(x, t, k) \exp \left(i k x \sigma_{3}\right)$. These functions are analytic in the complex $k$-plane cut along $\operatorname{Im}(k)=0$, they tend to the unit matrix as $k \rightarrow \infty$, and they have unit determinant. Actually $\Psi=\left(\Psi^{-}, \Psi^{+}\right)$, and the matrix $\Phi=\left(\Phi^{+}, \Phi^{-}\right)$is an entire function with respect to $k$. The eigenfunctions $\Phi$ and $\Psi$ satisfy certain symmetry conditions involving complex conjugation. We define the $2 \times 2$ matrix $\chi_{p}$ by

$\chi_{p}^{-}(x, t, k)=\left(\Psi^{-}(x, t, k), \Phi^{-}(x, t, k) \overline{\psi_{2}^{+}(0, t, \bar{k})}\right), \quad \chi_{p}^{+}(x, t, k)=\left(\Phi^{+}(x, t, k) / \psi_{2}^{+}(0, t, k), \Psi^{+}(x, t, k)\right)$,

for $k \in \mathbb{C}^{-}$, and $k \epsilon \mathbb{C}^{+}$respectively. The matrix $\chi_{p}$ has unit determinant and satisfies a certain jump condition

$$
\chi_{p}^{-}(x, t, k)=\chi_{p}^{+}(x, t, k) F(x, t, k), k \in \mathbb{R},
$$

where $F(x, t, k)$ is a $2 \times 2$ matrix involving $\psi_{1}^{+}(0, t, k)$ and $\psi_{2}^{+}(0, t, k)$.

(b) In 2.2 we use the $t$-part of the Lax pair to analyze $\psi(0, t, k)$. This function satisfies equation (1.3b) evaluated at $x=0$, where $C(t)=2 i k^{2} \sigma_{3}$. We define the $2 \times 2$ matrices $\hat{\Phi}(t, k)$ and $\hat{\Psi}(t, k)$ by the requirement that they satisfy the same equation as $\psi(0, t, k)$, and that $\hat{\Phi}(0, k)=I$, $\lim _{t \rightarrow \infty} \hat{\Psi}(t, k)=I$. These functions are analytic in the complex $k$-plane cut along $\operatorname{Im}\left(k^{2}\right)=0$, they tend to the unit matrix as $k \rightarrow \infty$, and they have unit determinant. Actually $\hat{\Psi}=\left(\hat{\Psi}^{-}, \hat{\Psi}^{+}\right)$, and the matrix $\hat{\Phi}=\left(\hat{\Phi}^{+}, \hat{\Phi}^{-}\right)$is an entire function with respect to $k$. The eigenfunctions $\hat{\Phi}$ and $\hat{\Psi}$ satisfy the same symmetry condition as those satisfied by $\Phi$ and $\Psi$. We define the matrix $\hat{Y}_{p}(t, k)$ by

$$
\left(\frac{\hat{\Phi}^{+}(t, k)}{s_{2}^{+}(k)}, \psi^{+}(0, t, k)\right),\left(\frac{\hat{\Psi}^{-}(t, k)}{\rho(k)}, \psi^{+}(0, t, k)\right),\left(\psi^{-}(0, t, k), \frac{\hat{\Psi}^{+}(t, k)}{\rho(\bar{k})}\right),\left(\psi^{-}(0, t, k), \frac{\hat{\Phi}^{-}(t, k)}{s_{2}^{+}(\bar{k})}\right),
$$

for $k \epsilon I, \ldots, I V$, where the scalars $s_{2}^{+}(k)$ and $\rho(k)$ are defined by 


$$
s_{2}^{+}(k) \doteqdot \psi_{2}^{+}(0,0, k), k \epsilon \mathbb{C}^{+} ; \rho(k) \doteqdot \hat{\Psi}_{1}^{-}(0, k) s_{2}^{+}(k)-\hat{\Psi}_{2}^{-}(0, k) s_{1}^{+}(k), k \epsilon I I ; s_{1}^{+}(k) \doteqdot \psi_{1}^{+}(0,0, k), k \epsilon \mathbb{C}^{+}
$$

The matrix $\hat{Y}_{p}$ has unit determinant and satisfies a certain jump condition

$$
\hat{Y}_{p}^{-}(t, k)=\hat{Y}_{p}^{+}(t, k) e^{-2 i k^{2} t \sigma_{3}} G(k) e^{2 i k^{2} t \sigma_{3}}, \quad k^{2} \in \mathbb{R},
$$

where the $2 \times 2$ matrix $G(k)$ involves the scalar $b(k)$ and $c(k)$ which are defined by

$$
b(k) \doteqdot \frac{s_{1}^{+}(k)}{s_{2}^{+}(k)}, \quad k \in \mathbb{R} ; \quad c(k) \doteqdot \frac{\hat{\Psi}_{2}^{-}(0, k)}{s_{2}^{+}(k) \rho(k)}, \quad k \in i \mathbb{R}^{+} \cup \mathbb{R}^{-}
$$

(c) In $\S 2.3$ we formulate a $\mathrm{RH}$ problem whose solution gives the solution of the initial-boudnary value problem of the NLS equation. Let the $2 \times 2$ matrix $\hat{Z}_{p}(x, t, k)$ be defined by

$$
\chi_{p}(x, t, k)\left(\begin{array}{cc}
1 & 0 \\
\frac{\left(\hat{Y}_{p}\right)_{21}(t, k)}{\left(\hat{Y}_{p}\right)_{22}(t, k)} e^{2 i k x} & 1
\end{array}\right), k \epsilon \mathbb{C}^{+} ; \quad \chi_{p}(x, t, k)\left(\begin{array}{cc}
1 & \frac{\left(\hat{Y}_{p}\right)_{12}(t, k)}{\left(\hat{Y}_{p}\right)_{11}(t, k)} e^{-2 i k x} \\
0 & 1
\end{array}\right), k \epsilon \mathbb{C}^{-}
$$

Then the meromorphic function $\hat{Z}_{p}(x, t, k)$, which has unit determinant, satisfies the RH problem

$$
\hat{Z}_{p}^{-}(x, t, k)=\hat{Z}_{p}^{+}(x, t, k) e^{-i\left(k x+2 k^{2} t\right) \sigma_{3}} G(k) e^{i\left(k x+2 k^{2} t\right) \sigma_{3}}, k^{2} \in \mathbb{R} ; \hat{Z} \rightarrow I \text { as } k \rightarrow \infty
$$

The jumps of this $\mathrm{RH}$ problem are given in Figure 1.1. The possible poles of $\hat{Z}_{p}(x, t, k)$ can occur only at the zeros of $\rho(k)$ for $k \epsilon I I$, and at the complex conjugates of these zeros. The additional conditions on the residues of $\hat{Z}_{p}$ have the usual "solitonic" form (equation (2.4.1) in section 2.4).

In section 3 we discuss rigorous aspects of the formalism developed in section 2. This includes finding conditions on $q(x, 0)$ and $q(0, t)$ which guarantee that:

(i) The linear integral equations for $\Phi(x, t, k)$ and $\Psi(x, t, k)$ are uniquely solvable for fixed $t$ and $k$.

(ii) The linear integral equations for $\hat{\Phi}(t, k)$ and $\hat{\Psi}(t, k)$ are uniquely solvable for fixed $k$.

(iii) The RH problem of Figure 1.1 is uniquely solvable.

The integral equations for $\Phi(x, t, k), \Psi(x, t, k)$ and for $\hat{\Phi}(t, k), \hat{\Psi}(t, k)$ are of the Volterra type; therefore they possess global, bounded, continuous solutions provided that $q(x, t)$ with $t$ fixed, and 
$\left\{q(0, t), q_{x}(0, t)\right\}$ belong in $L_{1}$. Furthermore under certain additional $L_{2}$ conditions, $\Phi(0, t, k)-I$, $\Psi(0, t, k)-I, \hat{\Phi}(t, k)-I$, and $\hat{\Psi}(t, k)-I \epsilon H_{1}$ for fixed $t$. This implies that the jump matrices of the RH problem of Figure 1.1 belong to $H_{1}$. Also, this $\mathrm{RH}$ problem satisfies a Schwartz-reflection symmetry. These two facts imply its unique solvability [6].

The most difficult part of the rigorous theory involves deriving $L_{1}$ estimates using PDE techniques. In section 3 we show how this can be achieved for $q(x, t)$ and $x q(x, t)$, where $t$ is fixed. The analogous but more difficult result for $q_{x}(0, t)$ is established in [13].

In section 4 we use the elegant approach of $[10]$ to study the asymptotic behavior of $q(x, t)$ as $t \rightarrow \infty$.

\section{The Formal Analysis}

We first study the $x$-RH problem.

\subsection{The $x$-Problem}

Let the $2 \times 2$ matrices $\varphi(x, t, k)$ and $\psi(x, t, k)$ be the solutions of equations (1.3) specified by the boundary conditions $\varphi(0, t, k)=I$ and $\lim _{x \rightarrow \infty} \exp \left(i k x \sigma_{3}\right) \psi(x, t, k)=I$, respectively. The matrix $\varphi(x, t, k)$ satisfies the Volterra integral equation

$$
\varphi(x, t, k)=e^{-i k x \sigma_{3}}+\int_{0}^{x} d \xi e^{-i k(x-\xi) \sigma_{3}} Q(\xi, t) \varphi(\xi, t, k) .
$$

The eigenfunction $\psi(x, t, k)$ satisfies a similar equation with $\int_{0}^{x}$ replaced by $-\int_{x}^{\infty}$. Letting

$$
\Phi(x, t, k)=\varphi(x, t, k) e^{i k x \sigma_{3}}, \quad \Psi(x, t, k)=\psi(x, t, k) e^{i k x \sigma_{3}},
$$

it follows that $\Phi$ satisfies the Volterra integral equation

$$
\Phi(x, t, k)=I+\int_{0}^{x} d \xi e^{-i k(x-\xi) \sigma_{3}} Q(\xi, t) \Phi(\xi, t, k) e^{i k(x-\xi) \sigma_{3}} .
$$

This equation, and the analogous equation satisfied by $\Psi(x, t, k)$, imply that

$$
\Phi=\left(\Phi^{+}, \Phi^{-}\right)=\left(\begin{array}{cc}
\Phi_{1}^{+} & \Phi_{1}^{-} \\
\Phi_{2}^{+} & \Phi_{2}^{-}
\end{array}\right), \Psi=\left(\Psi^{-}, \Psi^{+}\right)=\left(\begin{array}{cc}
\Psi_{1}^{-} & \Psi_{1}^{+} \\
\Psi_{2}^{-} & \Psi_{2}^{+}
\end{array}\right), \Phi, \Psi \rightarrow I \text { as } k \rightarrow \infty .
$$

This notation is explained in the introduction. It is important to notice, that since $\Phi$ satisfies a Volterra integral equation with compact support, $\Phi$ is an entire function with respect to $k$. 
The eigenfunctions $\Phi$ and $\Psi$ have unit determinant. Furthermore they satisfy certain symmetry conditions. Suppressing for convenience of writing the $x, t$ dependence, these symmetries are

$$
\overline{\Phi_{1}^{+}(k)}=\Phi_{2}^{-}(\bar{k}), \quad \overline{\Psi_{1}^{-}(k)}=\Psi_{2}^{+}(\bar{k}), \quad \overline{\Phi_{2}^{+}(k)}=\lambda \Phi_{1}^{-}(\bar{k}), \quad \overline{\Psi_{2}^{-}(k)}=\lambda \Psi_{1}^{+}(\bar{k}) .
$$

Since the functions $\varphi$ and $\psi$ are both solutions of equations (1.3), they are related through an $x$-independent matrix. Hence $\Phi=\Psi \exp \left(-i k x \sigma_{3}\right)(\psi(0, t, k))^{-1} \exp \left(i k x \sigma_{3}\right)$. Using this equation and defining $\chi_{p}$ by equation (1.12), one finds the jump condition (1.13), where the $2 \times 2$ matrix $F(x, t, k)$ is given by

$$
F_{11}=1, F_{22}=\frac{1}{\left|\psi_{2}^{+}(0, t, k)\right|^{2}}, F_{12}=\frac{-\psi_{1}^{+}(0, t, k)}{\overline{\psi_{2}^{+}(0, t, k)}} \exp (-2 i k x), F_{21}=\frac{\lambda \overline{\psi_{1}^{+}(0, t, k)}}{\psi_{2}^{+}(0, t, k)} \exp (2 i k x),
$$

and $\psi(0, t, k)=\Psi(0, t, k)$.

\subsection{The $t$-Problem}

The eigenfunction $\psi(x, t, k)$ satisfies equation (1.3b). Evaluating this equation as $x \rightarrow \infty$, it follows that $C(t)=2 i k^{2} \sigma_{3}$. Hence $\psi(0, t, k)$ solves

$$
\psi_{t}(0, t, k)+2 i k^{2}\left[\sigma_{3}, \psi(0, t, k)\right]=\hat{Q}(t, k) \psi(0, t, k)
$$

where

$$
\hat{Q}(t, k)=2 k Q(0, t)-i \lambda|q|^{2}(0, t) \sigma_{3}-i Q_{x}(0, t) \sigma_{3} .
$$

The matrix $\hat{Q}(t, k)$ involves $Q(0, t)$ which is known, but it also involves $Q_{x}(0, t)$ which is unknown. The main idea of [4] is to solve equation (2.2.1) by formulating an inverse scattering problem. The analysis is similar to that of $\S 2.1$. The eigenfunctions $\hat{\Phi}(t, k)$ and $\hat{\Psi}(t, k)$ are the solutions of equation (2.2.1) specified by the boundary conditions $\hat{\Phi}(0, k)=I$ and $\lim _{t \rightarrow \infty} \hat{\Psi}(t, k)=I$ respectively. In analogy with equation (2.1.3), $\hat{\Phi}$ satisfies the Volterra integral equation

$$
\hat{\Phi}(t, k)=I+\int_{0}^{t} d \tau e^{-2 i k^{2}(t-\tau) \sigma_{3}} \hat{Q}(\tau, k) \hat{\Phi}(\tau, k) e^{2 i k^{2}(t-\tau) \sigma_{3}} .
$$

This equation, and the analogous equation satisfied by $\hat{\Psi}(x, t, k)$ (where $\int_{0}^{t}$ is replaced by $-\int_{t}^{\infty}$ ) imply that 


$$
\hat{\Phi}=\left(\hat{\Phi}^{+}, \hat{\Phi}^{-}\right)=\left(\begin{array}{cc}
\hat{\Phi}_{1}^{+} & \hat{\Phi}_{1}^{-} \\
\hat{\Phi}_{2}^{+} & \hat{\Phi}_{2}^{-}
\end{array}\right), \quad \hat{\Psi}=\left(\hat{\Psi}^{-}, \hat{\Psi}^{+}\right)=\left(\begin{array}{cc}
\hat{\Psi}_{1}^{-} & \hat{\Psi}_{1}^{+} \\
\hat{\Psi}_{2}^{-} & \hat{\Psi}_{2}^{+}
\end{array}\right), \hat{\Phi}, \hat{\Psi} \rightarrow I \text { as } k \rightarrow \infty .
$$

This notation is explained in the introduction. The matrices $\hat{\Phi}$ and $\hat{\Psi}$ have unit determinant, and they satisfy the same symmetry relations as those satisfied by $\Phi$ and $\Psi$ (equations (2.1.5)).

It turns out that the following important relationships are valid between $\psi(0, t, k), \hat{\Psi}(t, k)$ and $\hat{\Phi}(t, k)$ :

$$
\begin{gathered}
\psi^{+}(0, t, k)=\frac{\psi_{2}^{+}(0,0, k)}{\hat{\Psi}_{2}^{+}(0, k)} \hat{\Psi}^{+}(t, k), k \epsilon I ; \psi^{-}(0, t, k)=\frac{\psi_{1}^{-}(0,0, k)}{\hat{\Psi}_{1}^{-}(0, k)} \hat{\Psi}^{-}(t, k), k \epsilon I V \\
\psi^{+}(0, t, k)=\psi_{2}^{+}(0,0, k) \hat{\Phi}^{-}(t, k)+\psi_{1}^{+}(0,0, k) \hat{\Phi}^{+}(t, k) \exp \left(-4 i k^{2} t\right), k \epsilon \mathbb{C}^{+}, \\
\psi^{-}(0, t, k)=\psi_{1}^{-}(0,0, k) \hat{\Phi}^{+}(t, k)+\psi_{2}^{-}(0,0, k) \hat{\Phi}^{-}(t, k) \exp \left(4 i k^{2} t\right), k \epsilon \mathbb{C}^{-},
\end{gathered}
$$

where $I$ and $I V$ denote the first and the fourth quadrants of the complex $k$-plane. To derive these equations we use the fact that $\psi^{-} E^{-1}, \psi^{+} E, \hat{\Psi}^{-} E^{-1}, \hat{\Phi}^{-} E, \hat{\Psi}^{+} E$, and $\hat{\Phi}^{+} E^{-1}$, where $E=$ $\exp \left(2 i k^{2} t\right)$, are related through equations involving constant coefficients. For example

$$
\psi^{+}(0, t, k)=\alpha \hat{\Psi}^{+}(t, k)+\beta \hat{\Psi}^{-}(t, k) \exp \left(-4 i k^{2} t\right), \quad k>0,
$$

where $\alpha$ and $\beta$ are $t$-independent scalars. Using $\beta=\operatorname{det}\left(\psi^{+}(0, t, k), \hat{\Psi}^{+}(t, k)\right) \exp \left(4 i k^{2} t\right)$, and the fact that the r.h.s. of this equation is a + function going to zero as $t \rightarrow \infty$, it follows that $\beta=0$. Then evaluating this equation at $t=0$ equation (2.2.5a) follows. Equations (2.2.5b), (2.2.6), (2.2.7) are derived in a similar way where one also uses the fact that $\hat{\Phi}$ is an entire function in the $k$-complex plane. We also note that equations (2.2.5b) and (2.2.7) follow from equations (2.2.5a) and (2.2.6) using the underline symmetry conditions.

Let us introduce the notation

$$
\psi(0) \doteqdot \psi(0,0, k), \quad \hat{\Psi}(0) \doteqdot \hat{\Psi}(0, k), \quad e=\exp \left(4 i k^{2} t\right)
$$

We define the $2 \times 2$ matrix $\hat{Y}_{p}$ by

$$
\left(\frac{\hat{\Phi}^{+}(t, k)}{\psi_{2}^{+}(0)}, \psi^{+}(0, t, k)\right),\left(\frac{\hat{\Psi}^{-}(t, k)}{\rho(k)}, \psi^{+}(0, t, k)\right),\left(\psi^{-}(0, t, k), \frac{\hat{\Psi}^{+}(t, k)}{\nu(k)}\right),\left(\psi^{-}(0, t, k), \frac{\hat{\Phi}^{-}(t, k)}{\psi_{1}^{-}(0)}\right),
$$


for $k \epsilon I, \ldots, I V$ respectively, where the scalar functions $\rho(k)$ and $\nu(k)$ are given by

$$
\rho(k) \doteqdot \hat{\Psi}_{1}^{-}(0) \psi_{2}^{+}(0)-\hat{\Psi}_{2}^{-}(0) \psi_{1}^{+}(0), \nu(k) \doteqdot \hat{\Psi}_{2}^{+}(0) \psi_{1}^{-}(0)-\hat{\Psi}_{1}^{+}(0) \psi_{2}^{-}(0) .
$$

In defining $\hat{Y}_{p}$, we use when it is possible $\psi(0, t, k)$ instead of $\hat{\Phi}(t, k)$ and $\hat{\Psi}(t, k)$; the scalars appearing in equation (2.2.9), are chosen by the requirement that $\operatorname{det} \hat{Y}_{p}=1$. Since the $\operatorname{det} \hat{Y}_{p}$ is $t$-independent, it follows that $\operatorname{det} \hat{Y}_{p}=1$ for $k \epsilon I I \cup I I I$. For $k \epsilon I$, the $\operatorname{det} \hat{Y}_{p}$ is given by

$$
\hat{\Phi}_{1}^{+} \frac{\psi_{2}^{+}}{\psi_{2}^{+}(0)}-\hat{\Phi}_{2}^{+} \frac{\psi_{1}^{+}}{\psi_{2}^{+}(0)}=\hat{\Phi}_{1}^{+} \frac{\hat{\Psi}_{2}^{+}}{\hat{\Psi}_{2}^{+}(0)}-\hat{\Phi}_{2}^{+} \frac{\hat{\Psi}_{1}^{+}}{\hat{\Psi}_{2}^{+}(0)},
$$

where we have used equation (2.2.5a). The r.h.s. of this equation simplifies to 1 using the 22 component of the equation $(\hat{\Phi})^{-1} \hat{\Psi}=\exp \left(-2 i k^{2} t \sigma_{3}\right) \hat{\Psi}(0) \exp \left(2 i k^{2} t \sigma_{3}\right)$, i.e. equation (2.2.11b),

$\hat{\Phi}_{2}^{-} \hat{\Psi}_{1}^{-}-\hat{\Phi}_{1}^{-} \hat{\Psi}_{2}^{-}=\hat{\Psi}_{1}^{-}(0), \hat{\Phi}_{1}^{+} \hat{\Psi}_{2}^{+}-\hat{\Phi}_{2}^{+} \hat{\Psi}_{1}^{+}=\hat{\Psi}_{2}^{+}(0), \hat{\Phi}_{2}^{-} \hat{\Psi}_{1}^{+}-\hat{\Phi}_{1}^{-} \hat{\Psi}_{2}^{+}=\hat{\Psi}_{1}^{+}(0) e^{-1}, \Phi_{1}^{+} \hat{\Psi}_{2}^{-}-\hat{\Phi}_{2}^{+} \hat{\Psi}_{1}^{-}=\hat{\Psi}_{2}^{-}(0) \epsilon$

A similar analysis implies that $\operatorname{det} \hat{Y}_{p}=1$ for $k \epsilon I V$.

The matrix $\hat{Y}_{p}$ satisfies the following jump conditions

$$
\begin{gathered}
\hat{Y}_{p}^{-}(t, k)=\hat{Y}_{p}^{+}(t, k) \hat{G}(t, k), \quad k^{2} \in \mathbb{R} . \\
k \epsilon i \mathbb{R}^{+}: \quad \hat{G}_{11}=\hat{G}_{22}=1, \hat{G}_{12}=0, \quad \hat{G}_{21}=\frac{\hat{\mathbf{\Psi}}_{2}^{-}(0)}{\psi_{2}^{+}(0) \rho} e . \\
k \epsilon i \mathbb{R}^{-}: \quad \hat{G}_{11}=\hat{G}_{12}=1, \hat{G}_{21}=0, \quad \hat{G}_{12}=-\frac{\left.\hat{\mathbf{\Psi}}_{1}^{+}(0)\right)}{\left(\psi_{1}^{-}(0)\right) \rho(k)} e^{-1} . \\
k \in \mathbb{R}^{+}: \hat{G}_{11}=1, \hat{G}_{22}=\frac{1}{\psi_{2}^{+}(0) \psi_{1}^{-}(0)}, \quad \hat{G}_{12}=-\frac{\psi_{1}^{+}(0)}{\psi_{1}^{-}(0)} e^{-1}, \hat{G}_{21}=\frac{\psi_{2}^{-}(0)}{\psi_{2}^{+}(0)} e . \\
k \in \mathbb{R}^{-}: \quad \hat{G}_{11}=1-R_{1} R_{2}, \hat{G}_{22}=1, \quad \hat{G}_{12}=-R_{1} e^{-1}, G_{21}=R_{2} e,
\end{gathered}
$$

where the scalar functions $R_{1}(k)$ and $R_{2}(k)$ are defined by

$$
R_{1}=\frac{\psi_{1}^{+}(0) \hat{\Psi}_{2}^{+}(0)-\psi_{2}^{+}(0) \hat{\Psi}_{1}^{+}(0)}{\psi_{2}^{-}(0) \hat{\Psi}_{1}^{+}(0)-\psi_{1}^{-}(0) \hat{\Psi}_{2}^{+}(0)}, \quad R_{2} \doteqdot \frac{\psi_{1}^{-}(0) \hat{\Psi}_{2}^{-}(0)-\psi_{2}^{-}(0) \hat{\Psi}_{1}^{-}(0)}{\hat{\Psi}_{1}^{-}(0) \psi_{2}^{+}(0)-\hat{\Psi}_{2}^{-}(0) \psi_{1}^{+}(0)}
$$

We now indicate how these jump conditions can be derived. 
$k \epsilon R^{+}$: Substituting the expression for $\hat{Y}_{p}$ in equation $(2.2 .12)$, and using $\operatorname{det} \psi=\operatorname{det} \hat{\Phi}=1$, one finds $\hat{G}_{11}=1, \hat{G}_{22}=1 / \psi_{2}^{+}(0) \psi_{1}^{-}(0)$, and

$$
\hat{G}_{12}=\hat{\Phi}_{1}^{-} \frac{\psi_{2}^{+}}{\psi_{1}^{-}(0)}-\hat{\Phi}_{2}^{-} \frac{\psi_{1}^{+}}{\psi_{1}^{-}(0)}, \quad \hat{G}_{21}=\hat{\Phi}_{1}^{+} \frac{\psi_{2}^{-}}{\psi_{2}^{+}(0)}-\hat{\Phi}_{2}^{+} \frac{\psi_{1}^{-}}{\psi_{2}^{+}(0)}
$$

Using equations $(2.2 .5)$ to eliminate $\psi_{2}^{+}, \psi_{1}^{+}, \psi_{2}^{-}, \psi_{1}^{-}$, and then using (2.2.11c) and (2.2.11d), these equations become

$$
\hat{G}_{12}=-\frac{\psi_{2}^{+}(0) \hat{\Psi}_{1}^{+}(0)}{\psi_{1}^{-}(0) \hat{\Psi}_{2}^{+}(0)} e^{-1}, \quad \hat{G}_{21}=\frac{\psi_{1}^{-}(0) \hat{\Psi}_{2}^{-}(0)}{\psi_{2}^{+}(0) \hat{\Psi}_{1}^{-}(0)} e .
$$

Using equation (2.2.5) evaluated at $t=0$, these expressions yield the expression for $\hat{G}_{12}$ and $\hat{G}_{21}$ given in equation (2.2.15).

$k \epsilon i R^{+}:$Substituting the expressions for $\hat{Y}_{p}$ in equation $(2.2 .12)$, one finds $\hat{G}_{11}=1, \hat{G}_{12}=0$, and

$$
\hat{G}_{22}=\hat{\Phi}_{1}^{+} \frac{\psi_{2}^{+}}{\psi_{2}^{+}(0)}-\hat{\Phi}_{2}^{+} \frac{\psi_{1}^{+}}{\psi_{2}^{+}(0)}, \quad \hat{G}_{21}=\frac{\hat{\Phi}_{1}^{+} \hat{\Psi}_{2}^{-}-\hat{\Phi}_{2}^{+} \hat{\Psi}_{1}^{-}}{\rho \psi_{2}^{+}(0)}
$$

Using equation (2.2.5a) to eliminate $\psi_{2}^{+}$and $\psi_{1}^{+}$, and then using equation $(2.2 .11 \mathrm{~b})$ it follows that $\hat{G}_{22}=1$. Using equation $(2.2 .11 \mathrm{~d})$, it follows that $\hat{G}_{21}=\hat{\Psi}_{2}^{-}(0) e / \rho \psi_{2}^{+}(0)$.

$k \epsilon i R^{-}, k \epsilon R^{-}:$the derivation of the jump matrices defined in equations (2.2.14) and (2.2.16) is similar to the above and hence is omitted.

The number of independent functions appearing in the above jumps can be reduced by using the underline symmetry conditions. Some of these functions can be computed effectively in terms of initial data, while the rest, although depend in a nonlinear way on the boundary data, can be given an effective characterization in the $k$-complex plane.

Let us summarize the main formal results of this subsection.

Proposition 2.1 Let the $2 \times 2$ matrix value function $\hat{Y}_{p}(t, k)$ be defined by equation (1.14). Then $\hat{Y}_{p}$ satisfies equation (1.16), where the jump matrices are depicted in Figure 1.1 with $\theta$ replaced by $4 i k^{2} t$.

The functions $s_{1}^{+}(k)$ and $s_{2}^{+}(k)$ defined in (1.15) can be computed in terms of the initial data $q(x, 0)$. The function $c(k)$ defined in (1.17) depends in a nonlinear way on the boundary data $q(0, t)$. These functions, which are called the scattering data, have the following properties: 
1. $s_{1}^{+}(k)$ and $s_{2}^{+}(k)$ are analytic for $k \in \mathbb{C}^{+} ; s_{1}^{+}(k) \rightarrow 0$, and $s_{2}^{+}(k) \rightarrow 1$, as $k \rightarrow \infty$.

2.

$$
\left|s_{2}^{+}(k)\right|^{2}-\lambda\left|s_{1}^{+}(k)\right|^{2}=1, \quad k \in \mathbb{R} .
$$

3. $c(k)$ is analytic in $I I$ except for possible poles, which can occur at the zeros of $s_{2}^{+}(k)$ and at the points $\left\{k_{j}\right\}_{1}^{N}, k_{j} \epsilon I I$ which are the zeros of the function $\rho(k)$ defined in $(1.15) ; c(k) \rightarrow 0$ as $k \rightarrow \infty$.

\section{Proof}

It is straightforward to derive the jump matrices appearing in Figure 1.1 from equations (2.2.13)(2.2.16): The jumps on $R^{+}, i \mathbb{R}^{+}$follow from the notations introduced in (1.15), (1.17), the underline symmetry, and the relation $\left|s_{2}^{+}\right|^{-2}=1-\lambda|b|^{2}$, which in turn follows from $\operatorname{det} \psi(0)=1$ and the underline symmetry. The jump on $i R^{-}$follows from the underline symmetry. Taking again into account the underline symmetry and the relation $\operatorname{det} \psi(0)=1$, it follows that for $k \epsilon \mathbb{R}^{-}$,

$$
\begin{gathered}
b(k)-\lambda \overline{c(k)}=\frac{\psi_{1}^{+}(0)}{\psi_{1}^{-}(0)}-\frac{\hat{\Psi}_{1}^{+}(0)}{\psi_{1}^{-}(0)\left[\hat{\Psi}_{2}^{+}(0) \psi_{1}^{-}(0)-\hat{\Psi}_{1}^{+}(0) \psi_{2}^{-}(0)\right]}= \\
\frac{\psi_{1}^{+}(0) \hat{\Psi}_{2}^{+}(0) \psi_{1}^{-}(0)-\hat{\Psi}_{1}^{+}(0)\left(1+\psi_{1}^{+}(0) \psi_{2}^{-}(0)\right)}{\psi_{1}^{-}(0)\left[\hat{\Psi}_{2}^{+}(0) \psi_{1}^{-}(0)-\hat{\Psi}_{1}^{+}(0) \psi_{2}^{-}(0)\right]}=\frac{\psi_{1}^{+}(0) \hat{\Psi}_{2}^{+}(0)-\hat{\Psi}_{1}^{+}(0) \psi_{2}^{+}(0)}{\hat{\Psi}_{2}^{+}(0) \psi_{1}^{-}(0)-\hat{\Psi}_{1}^{+}(0) \psi_{2}^{-}(0)}=-R_{1} .
\end{gathered}
$$

Since $R_{2}=\lambda \bar{R}_{1}$, this implies the jump on $\mathbb{R}^{-}$indicated in Figure 1.1.

The initial data $q(x, 0)$ can be used to compute $\psi(x, 0, k)$, which in turn implies $\psi(0,0, k)$. Hence all of the components of $\psi(0,0, k)$ can be effectively calculated in terms of $q(x, 0)$. Equation (2.2.18) follows from $\operatorname{det} \psi(0)=1$. Although the scattering data $c(k)$ depends in a nonlinear way on the boundary data $q(0, t)$, its analytic structure can be characterized explicitly. The large $k$ behavior of $s_{1}^{+}, s_{2}^{+}$, and $c(k)$ follows from the large $k$ behavior of $\psi_{1}^{+}(0,0, k), \psi_{2}^{+}(0,0, k), \hat{\Psi}_{2}^{-}$, and $\hat{\Psi}_{1}^{-}$. The possible poles for $c(k)$ appear at the possible zeros of $s_{2}^{+}(k)$ and of $\rho(k)$. This completes the proof of the proposition 2.1 .

Remark If one investigates the direct and inverse problems associated with equation (1.3b), without assuming a relationship between $q(0, t)$ and $q_{x}(0, t)$, one finds that the basic scattering data is the reflection coefficient $r(k)$ defined by 


$$
r(k)=\frac{\hat{\Psi}_{1}^{+}(0, k)}{\hat{\Psi}_{2}^{+}(0, k)} .
$$

The only analytic restriction on $r(k)$ is that it is meromorphic in $I \cup I I I$. However, in our case, since $q(0, t)$ and $q_{x}(0, t)$ are the boundary values of the NLS equation, there exists a relationship between $q(0, t)$ and $q_{x}(0, t)$. It is quite interesting that although this relationship is highly nonlinear, its representation in the scattering space is very simple: $r(k)$ has analytic continuation for $k \epsilon I I$. Indeed, equation $(2.2 .5 \mathrm{a}$ ) evaluated at $t=0$ becomes

$$
\frac{\psi_{1}^{+}(0,0, k)}{\psi_{2}^{+}(0,0, k)}=\frac{\hat{\Psi}_{1}^{+}(0, k)}{\hat{\Psi}_{2}^{+}(0, k)}, k \in I .
$$

But $\psi^{+}(0,0, k)$ is analytic in $I \cup I I$, hence $r(k)$ has an analytic continuation across $i \mathbb{R}^{+}$. The function $c(k)$ is related to $r(k)$ through the equation

$$
c(k)=\frac{\lambda \overline{r(\bar{k})}}{\left(s_{2}^{+}(k)\right)^{2}\left(1-\lambda \frac{s_{1}^{+}(k)}{s_{2}^{+}(k)} \overline{r(\bar{k})}\right)} .
$$

\subsection{The RH Problem}

In this section we formulate a $\mathrm{RH}$ problem, whose solution gives the solution of the initial boundary value problem of the NLS equation.

Proposition 2.2 Let the $2 \times 2$ matrix $\hat{Z}_{p}(x, t, k)$ be defined by equation (1.18). Then:

(i) $\hat{Z}_{p}(x, t, k)$ has unit determinant and it satisfies the $\mathrm{RH}$ problem (1.19).

(ii) $\hat{Z}_{p}(x, t, k)$ is a meromorphic function in the complex $k$-plane cut along $\operatorname{Im}\left(k^{2}\right)=0$. Its possible poles can occur only at the zeros of $\rho(k)$ for $k \epsilon I I$, and at the complex conjugate of these zeros.

(iii) The eigenfunction $\Psi^{+}(x, t, k)$ and the potential $q(x, t)$ can be obtained from $\hat{Z}_{p}(x, t, k)$ by

$$
\Psi^{+}(x, t, k)=\hat{Z}_{p}(x, t, k)(0,1)^{T}, k \epsilon \mathbb{C}^{+} ; \quad q(x, t)=2 i \lim _{k \rightarrow \infty}\left(k \hat{Z}_{p}(x, t, k)\right)_{12}, \quad k \epsilon I,
$$

where the subscript 12 denotes the 12 components of $\hat{Z}_{p}$. 
Proof For convenience of writing we drop the subscript $p$ in $\hat{Y}$. (i) We first note that the jump matrix $F(x, t, k)$ defined in equation (2.1.6) can be written as

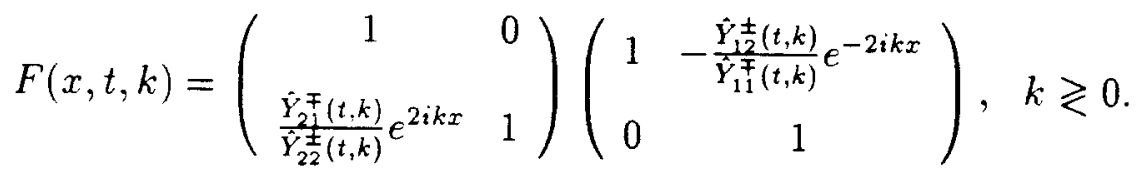

Consider now the jump conditions for $\hat{Z}_{p}(x, t, k)$.

$k \in \mathbb{R}^{+}:$Equations (1.18), (1.13), and (2.3.2) yield

$$
\begin{aligned}
& {\left[\hat{Z}_{p}^{+}(x, t, k)\right]^{-1} \hat{Z}_{p}^{-}(x, t, k)=\left(\begin{array}{cc}
1 & 0 \\
-\frac{\hat{Y}_{2}^{+}}{\hat{Y}_{22}^{+}} e^{2 i k x} & 1
\end{array}\right) F(x, t, k)\left(\begin{array}{cc}
1 & \frac{\hat{Y}_{12}^{-}}{\hat{Y}_{11}^{-}} e^{-2 i k x} \\
0 & 1
\end{array}\right)=} \\
& =\left(\begin{array}{cc}
1 & 0 \\
\frac{\hat{Y}_{21}^{-}-\hat{Y}_{21}^{+}}{\hat{Y}_{22}^{+}} e^{2 i k x} & 1
\end{array}\right)\left(\begin{array}{cc}
1 & \frac{\hat{Y}_{12}^{-}-\hat{Y}_{12}^{+}}{\hat{Y}_{11}^{-}} e^{-2 i k x} \\
0 & 1
\end{array}\right)
\end{aligned}
$$

where $\hat{Y}_{j l}^{ \pm} \equiv \hat{Y}_{j l}^{ \pm}(t, k)$. Equation (1.16) implies

$$
\hat{Y}_{21}^{-}-\hat{Y}_{21}^{+}=\hat{Y}_{22}^{+} \hat{G}_{21}(t, k), \quad \hat{Y}_{12}^{-}-\hat{Y}_{12}^{+}=\hat{Y}_{11}^{-} \hat{G}_{12}(t, k) .
$$

Using these equations it follows that

$\left[\hat{Z}_{p}^{+}(x, t, k)\right]^{-1} \hat{Z}_{p}^{-}(x, t, k)=\left(\begin{array}{cc}1 & 0 \\ \hat{G}_{21}(t, k) e^{2 i k x} & 1\end{array}\right)\left(\begin{array}{cc}1 & \hat{G}_{12}(t, k) e^{-2 i k x} \\ 0 & 1\end{array}\right)=e^{-i\left(k x+2 k^{2} t\right) \sigma_{3}} G(k) e^{i\left(k x+2 k^{2} t\right) \sigma_{3}}$

$k \epsilon i \mathbb{R}^{+}$: Equation (1.18) yields

$$
\left[\hat{Y}^{+}(x, t, k)\right]^{-1} \hat{Y}^{-}(x, t, k)=\left(\begin{array}{ccc}
1 & 0 \\
\left(\begin{array}{cc}
\hat{Y}_{21}^{-} \\
\hat{Y}_{22}^{-}
\end{array}-\frac{\hat{Y}_{21}^{+}}{\hat{Y}_{22}^{+}}\right) e^{2 i k x} & 1
\end{array}\right)
$$

Equation (1.16) implies

$$
\hat{Y}_{22}^{-}=\hat{Y}_{22}^{+}, \quad \hat{Y}_{21}^{-}-\hat{Y}_{21}^{+}=\hat{Y}_{22}^{+} \hat{G}_{21}(t, k) .
$$

Using these equations it follows that

$$
\left[\hat{Y}^{+}(x, t, k)\right]^{-1} \hat{Y}^{-}(x, t, k)=\left(\begin{array}{cc}
1 & 0 \\
\hat{G}_{21}(t, k) e^{2 i k x} & 1
\end{array}\right)=e^{-i\left(k x+2 k^{2} t\right) \sigma_{3}} G(k) e^{i\left(k x+2 k^{2} t\right) \sigma_{3}}
$$


The derivation of the jump conditions for $k \in \mathbb{R}^{-}$and $k \epsilon i \mathbb{R}^{-}$is similar to the above and hence is omitted.

(ii) Let $k \epsilon I$. Using the definitions of $\chi_{p}(x, t, k)$ and $\hat{Y}_{p}(t, k)$ for $k \epsilon I$, i.e.

$$
\chi_{p}(x, t, k)=\left(\frac{\Phi^{+}(x, t, k)}{\psi_{2}^{+}(0, t, k)}, \Psi^{+}(x, t, k)\right), \quad \hat{Y}_{21}(t, k)=\frac{\hat{\Phi}_{2}^{+}(t, k)}{s_{2}^{+}(k)}, \quad \hat{Y}_{22}(t, k)=\psi_{2}^{+}(0, t, k),
$$

equation (1.18) yields

$$
\hat{Z}_{p}(x, t, k)=\left(\frac{1}{\psi_{2}^{+}(0, t, k)}\left(\Phi^{+}(x, t, k)+\frac{\hat{\Phi}_{2}^{+}(t, k)}{s_{2}^{+}(k)} \Psi^{+}(x, t, k) e^{2 i k x}\right), \quad \Psi^{+}(x, t, k)\right), k \epsilon I .
$$

We will show that at the possible zeros of $\psi_{2}^{+}(0, t, k)$, denoted by $k_{0}$,

$$
\Phi^{+}\left(x, t, k_{0}\right)+\frac{\hat{\Phi}_{2}^{+}\left(t, k_{0}\right)}{s_{2}^{+}\left(k_{0}\right)} \Psi^{+}\left(x, t, k_{0}\right) e^{2 i k_{0} x}=0, \quad k_{0} \epsilon I
$$

hence the possible poles of $\hat{Z}_{p}(x, t, k)$ for $k \epsilon I$, can occur only at the fixed possible zeros of $s_{2}^{+}(k)$.

To derive equation (2.3.4) we first relate $\Phi^{+}(x, t, k)$ to $\Psi^{+}(x, t, k) e^{2 i k x}$, and to $\Phi^{-}(x, t, k) e^{2 i k x}$ through an equation with $x$-independent coefficients. Evaluating this equation at $x=0$ it follows that

$$
\Phi^{+}(x, t, k)=\frac{\Psi^{+}(x, t, k)}{\psi_{1}^{+}(0, t, k)} e^{2 i k x}-\frac{\psi_{2}^{+}(0, t, k)}{\psi_{1}^{+}(0, t, k)} \Phi^{-}(x, t, k) e^{2 i k x}, k \in \mathbb{C}^{+} .
$$

Equation (2.3.4) follow's from equation (2.3.5) evaluated at $k=k_{0}$. Indeed, when $k=k_{0}$, $\psi_{2}^{+}\left(0, t, k_{0}\right)=0$, hence equation $(2.2 .6)$ yields

$$
\begin{gathered}
\psi_{1}^{+}\left(0, t, k_{0}\right)=s_{2}^{+}\left(k_{0}\right) \hat{\Phi}_{1}^{-}\left(t, k_{0}\right)+s_{1}^{+}\left(k_{0}\right) \hat{\Phi}_{1}^{+}\left(t, k_{0}\right) e^{-4 i k_{0}^{2} t} \\
0=s_{2}^{+}\left(k_{0}\right) \hat{\Phi}_{2}^{-}\left(t, k_{0}\right)+s_{1}^{+}\left(k_{0}\right) \hat{\Phi}_{2}^{+}\left(t, k_{0}\right) e^{-4 i k_{0}^{2} t}, \quad k_{0} \epsilon I
\end{gathered}
$$

These equations together with $\operatorname{det} \hat{\Phi}=1$ imply $\psi_{1}^{+}\left(0, t, k_{0}\right) \hat{\Phi}_{2}^{+}\left(t, k_{0}\right)=-s_{2}^{+}\left(k_{0}\right)$.

Let $k \epsilon I I$. Instead of equation (2.3.3) we now find

$$
\hat{Z}_{p}(x, t, k)=\left(\frac{1}{\psi_{2}^{+}(0, t, k)}\left(\Phi^{+}(x, t, k)+\frac{\hat{\Psi}_{2}^{-}(t, k)}{\rho(k)} \Psi^{+}(x, t, k) e^{2 i k x}\right), \quad \Psi^{+}(x, t, k)\right), \quad k \epsilon I I
$$


Again it can be shown that if $\psi_{2}^{+}\left(0, t, k_{0}\right)=0, k_{0} \epsilon I I$, then

$$
\Phi^{+}\left(x, t, k_{0}\right)+\frac{\hat{\Psi}_{2}^{-}\left(t, k_{0}\right)}{\rho\left(k_{0}\right)} \Psi^{+}\left(x, t, k_{0}\right) e^{2 i k_{0} x}=0, \quad k_{0} \epsilon I I,
$$

hence the possible poles of $\hat{Z}_{p}(x, t, k)$ for $k c I I$, can occur only at the fixed possible zeros of $\rho(k)$. Equation (2.3.8) follows from equation (2.3.5) using $\operatorname{det} \hat{Y}_{p}=1, k \epsilon I I$, i.e.

$$
\rho(k)=\hat{\Psi}_{1}^{-}(t, k) \psi_{2}^{+}(0, t, k)-\hat{\Psi}_{2}^{-}(t, k) \psi_{1}^{+}(0, t, k) .
$$

The absence of singularities of $\hat{Z}_{p}(x, t, k)$ at the possible zeros of $\psi_{1}^{-}(0, t, k)$ can be proven in a similar manner; alternatively one can use the underline symmetry.

To conclude the proof of statement (ii) it remains to notice that $s_{2}^{+}(k)$ cannot have zeros in $I$. In fact, if $s_{2}^{+}\left(k_{0}\right)=0$ for $k_{0} \epsilon I$, equation (2.2.6) implies

$$
e^{4 i k_{0}^{2} t} \psi^{+}\left(0, t, k_{0}\right)=s_{1}^{+}\left(k_{0}\right) \hat{\Phi}^{+}\left(t, k_{0}\right), \quad k_{0} \epsilon I
$$

This means

$$
\hat{\Phi}^{+}\left(t, k_{0}\right) \rightarrow 0, \quad t \rightarrow+\infty
$$

which contradicts $\operatorname{det} \hat{\Phi}=1$ for all $t>0$. Taking into account that $s_{2}^{+}(k)$ participates in the definition of $\hat{Z}_{p}$ only in the first quadrant $I$, we conclude that the function $\hat{Z}_{p}(x, t, k)$ can have poles only in the quadrants $I I$ and $I I I$, and that these poles occur at the zeros of $\rho(k)$ and $\overline{\rho(\bar{k})}$ respectively. As we will see in section 4, this fact implies that the possible solitons of the initialboundary value problem (1.1) move away from the boundary.

(iii) Equation (2.3.1b) follows from the large $k$ asymptotics of equation (1.3).

We conclude this subsection with a discussion of the appropriate data needed to solve the $\mathrm{RH}$ problem.

Proposition 2.3 The RH problem for $\hat{Z}_{p}$ is uniquely specified by the following data, called scattering data: (i) $c(k)$ for $k \in \mathbb{R}^{-} \cup i \mathbb{R}^{+}$; (ii) $s_{1}^{+}(k)$ for $k \in \mathbb{R}$; (iii) the zeros of $s_{2}^{+}(k)$ for $k \epsilon \mathbb{C}^{+}$; (iv) the zeros $\left\{k_{j}\right\}_{1}^{N}$ of $\rho(k)$ and the residues $\left\{c_{j}\right\}_{1}^{N}$ of $c(k)$ at these poles. 
Proof Indeed, (ii) and (iii) together with the equation (2.2.18) yield $s_{2}^{+}$through the solution of a scalar RH problem. Then, $c(k), s_{1}^{+}$, and $s_{2}^{+}$specify all the jump conditions. The possible poles of $\hat{Z}_{p}$ can occur at the possible zeros of $\rho(k)$. The additional conditions on the residues of $\hat{Z}_{p}$ are given in the next section (equation 2.4.1)).

It is worth mentioning that $\rho(k) \neq 0$ for $k \epsilon i \mathbb{R}^{+}$. Indeed, equation $(2.2 .5 \mathrm{a})$ implies that for $k \epsilon i \mathbb{R}^{+}$ $s_{1}^{+}(k)=\rho(k) \hat{\Psi}_{1}^{+}(0, k)$ and $s_{2}^{+}(k)=\rho(k) \hat{\Psi}_{2}^{+}(0, k)$. Thus $\rho(k) \neq 0$ since $s_{1}^{+}$and $s_{2}^{+}$cannot vanish simultaneously.

Remark 1 The above analysis is valid for the generic situation, i.e. we assume that all zeros of $\psi_{2}^{+}(0, t, k)$, of $s_{2}^{+}(k)$, and of $\rho(k)$ are simple, they do not coincide with each other, they do not lie on the cross $I m k^{2}=0$, and there is a finite number of them.

Remark 2 The function $c(k), k \in \mathbb{R}^{-} \cup i \mathbb{R}^{+}$, satisfies certain restrictions. Indeed, since it is the boundary value of a function meromorphic in $I I$ and decreasing as $k \rightarrow \infty, c(k)$ satisfies the following infinite set of conditions,

$$
\frac{1}{2 \pi i} \int_{\mathbb{R}-\cup i \mathbb{R}} \frac{c\left(k^{\prime}\right)}{k^{\prime}-k} d k^{\prime}=\sum_{j=1}^{N} \frac{c_{j}}{k_{j}-k}+\sum_{j=1}^{M} \frac{c_{j}^{+}}{k_{j}^{+}-k}, \quad \forall k \notin I I
$$

or

$$
\frac{1}{2 \pi i} \int_{\mathbb{R}^{-}-\mathbb{U}^{+}} c(k)(k-1)^{-n} d k=\sum_{j=1}^{N} c_{j}\left(k_{j}-1\right)^{-n}+\sum_{j=1}^{M} c_{j}^{+}\left(k_{j}^{+}-1\right)^{-n}
$$

where $k_{j}^{+}, j=1 \ldots M$ are the zeros of $s_{2}^{+}(k)$, and $c_{j}^{+}$are the corresponding residues of $c(k)$. As a set of independent parameters for $c(k)$ one can take the whole set of its poles and the corresponding residues $\left\{k_{j}, k_{j}^{+}, c_{j}, c_{j}^{+}\right\}$supplemented by

$$
\alpha(k) \doteqdot R e\left\{c(k)-\sum_{j=1}^{N} \frac{c_{j}}{k-k_{j}}-\sum_{j=1}^{M} \frac{c_{j}^{+}}{k-k_{j}^{+}}\right\}, \quad k \in \mathbb{R}^{-} \cup i \mathbb{R}^{+} .
$$

Having $\left\{\alpha(k) ; k_{j}, k_{j}^{+}, c_{j}, c_{j}^{+}\right\}$one can reconstruct $c(k)$ in closed form. Also, in order to reconstruct $s_{1}^{+}(k)$, one needs to know $\beta(k) \doteqdot R e s_{1}^{+}(k)$. Thus the set of independent parameters for our $\mathrm{RH}$ problem are

$$
\left\{\alpha(k) ; \beta(k) ; k_{j}, k_{j}^{+}, c_{j}, c_{j}^{+}\right\}
$$


where $\alpha(k)$ and $\beta(k)$ are real-valued decreasing functions defined on the whole line, $k_{j}, k_{j}^{+} \epsilon I I, c_{j}$, $c_{j}^{+} \epsilon \mathbb{C} \backslash\{0\}$. In particular, this means that the functional dimension of the set can be represented by two complex valued functions defined on the half-line.

\subsection{Solitons}

It turns out that the zeros of $\rho(k)$ give rise to solitons.

Proposition 2.4 Assume that $\rho(k)$ has a finite number of simple zeros for $k \epsilon I I$. Let these zeros be denoted by $\left\{k_{j}\right\}_{1}^{N}$. Let $\left\{c_{j}\right\}_{1}^{N}$ be the corresponding residues of $c(k)$. Then:

(i) The first column $\hat{Z}_{p}^{(1)}(x, t, k)$ of $\hat{Z}_{p}(x, t, k)$ has a simple pole at $k_{j}, j=1, \ldots, N$ and the second column $\hat{Z}_{p}^{(2)}(x, t, k)$ of $\hat{Z}_{p}(x, t, k)$ has a simple pole at $\bar{k}_{j}, j=1, \ldots, N$; the corresponding residues satisfy the equations

$$
\begin{aligned}
& \operatorname{res} \hat{Z}_{p}^{(1)}(x, t, k)=c_{j} e^{\theta\left(k_{j}\right)} \hat{Z}_{p}^{(2)}\left(x, t, k_{j}\right), \quad \theta(k) \doteqdot 2 i\left(k x+2 k^{2} t\right), \\
& k_{j} \\
& \frac{\operatorname{res}}{k_{j}} \hat{Z}_{p}^{(2)}(x, t, k)=\lambda \bar{c}_{j} e^{-\theta\left(\bar{k}_{j}\right)} \hat{Z}_{p}^{(1)}\left(x, t, \bar{k}_{j}\right)
\end{aligned}
$$

(ii) Equations (2.4.1) together with the jump condition (1.19a), and the asymptotic condition (1.19b), characterize the function $\hat{Z}_{p}(x, t, k)$ uniquely.

(iii) The RH problem (1.19), (2.4.1) can be solved as follows: Let $\hat{Z}(x, t, k)$ be the solution of a $\mathrm{RH}$ problem satisfying the same jump conditions as $\hat{Z}_{p}(x, t, k)$, but with $c(k)$ and $b(k)$ replaced by $c_{0}(k) \doteqdot c(k) \Pi_{j=1}^{N}\left(k-k_{j}\right) /\left(k-\bar{k}_{j}\right)$ and $b_{0}(k)=b(k) \Pi_{j=1}^{N}\left(k-\bar{k}_{j}\right) /\left(k-k_{j}\right)$. This RH problem is regular, i.e. $\hat{Z}(x, t, k)$ has no singularities in the complex $k$-plane. Then $\hat{Z}_{p}(x, t, k)$ can be found from $\hat{Z}$ through the equation

$$
\hat{Z}_{p}(x, t, k)=\left(k I+B_{N}\right)\left(k I+B_{N-1}\right) \ldots\left(k I+B_{1}\right) \hat{Z}(x, t, k)\left(\begin{array}{cc}
\frac{1}{\Pi_{j=1}^{N}\left(k-k_{j}\right)} & 0 \\
0 & \frac{1}{\Pi_{j=1}^{N}\left(k-k_{j}\right)}
\end{array}\right)
$$

where the $2 \times 2$ matrices $B_{1}, \ldots, B_{N}$ are independent of $k$. These matrices can be determined recursively by solving the algebraic equations 


$$
\left(k_{j} I+B_{j}\right) \hat{Z}_{j-1}\left(x, t, k_{j}\right)\left(\begin{array}{c}
1 \\
-d_{j}(x, t)
\end{array}\right)=0, \quad\left(\bar{k}_{j} I+B_{j}\right) \hat{Z}_{j-1}\left(x, t, \bar{k}_{j}\right)\left(\begin{array}{c}
-\lambda \bar{d}_{j}(x, t) \\
1
\end{array}\right)=0
$$

where

$$
\begin{gathered}
\hat{Z}_{j}(x, t, k)=\left(k I+B_{j}\right) \hat{Z}_{j-1}(x, t, k), \quad j=1, \ldots, N-1, \quad \hat{Z}_{0}=\hat{Z}, \\
d_{j}(x, t)=c_{j} \frac{\prod_{l=1, l \neq j}^{N}\left(k_{j}-k_{l}\right)}{\prod_{l=1}^{N}\left(k_{j}-\bar{k}_{l}\right)} \exp \left(2 i k_{j} x+4 i k_{j}^{2} t\right) .
\end{gathered}
$$

The RH problem for $\hat{Z}$, and the algebraic equations for $B_{1}, \ldots, B_{N}$ in the focusing case $\lambda=-1$, are always solvable.

Proof $\hat{Z}_{p}(x, t, k)$ is given by equations (2.3.3) and (2.3.7) for $k$ in the first and second quadrants respectively. Since $s_{2}^{+}(k)$ has no zeros in $I, \hat{Z}_{p}$ is analytic for $k \epsilon I$, while the first column of $\hat{Z}_{p}$ has poles for $k \epsilon I I$ at the zeros of $\rho(k)$, i.e. at the points $k_{1}, \ldots, k_{N}$, which are simultaneously the poles of $c(k)$. For $k \epsilon i \mathbb{R}^{+}$,

$$
\hat{Z}_{p}^{-}(x, t, k)\left(\begin{array}{cc}
1 & 0 \\
-c(k) e^{\theta} & 1
\end{array}\right)=\hat{Z}_{p}^{+}(x, t, k) .
$$

Because $\hat{\Phi}(t, k)$ is an entire function, both sides of equation (2.4.5) have an analytic continuation into the second quadrant of the complex $k$-plane; moreover, $\hat{Z}_{p}^{+}(x, t, k)$ has no singularities at $k_{1}, \ldots, k_{N}$. This shows that the l.h.s. of equation $(2.4 .5)$ has no singularities at $k_{1}, \ldots, k_{N}$ as well. Similar considerations apply for $k \epsilon I I I$, thus

$$
\hat{Z}_{p}(x, t, k)\left(\begin{array}{c}
1 \\
-c(k) e^{\theta}
\end{array}\right) \text { and } \hat{Z}_{p}(x, t, k)\left(\begin{array}{c}
-\lambda \overline{c(\bar{k})} e^{-\theta(k)} \\
1
\end{array}\right)
$$

have no singularities at $k_{j}$ and $\bar{k}_{j}$ respectively. This implies equations (2.4.1).

In order to prove statement (ii), we suppose that $\hat{z}_{p}(x, t, k)$ is another solution of the RH problem (1.19), (2.4.1). The function $\hat{R}(k)$,

$$
\hat{R}=\hat{z}_{p} \hat{Z}_{p}^{-1}
$$

has no jumps at $k^{2} \epsilon \mathbb{R}$. Its only possible singularities $\left(\operatorname{det} \hat{Z}_{p}=1\right)$ are at the points $k_{j}$ and $\bar{k}_{j}$. For $k \epsilon I I$ one can rewrite $\hat{R}$ as 


$$
\left.\hat{R}_{k}\right)=\hat{z}_{p}(k)\left(\begin{array}{cc}
1 & 0 \\
-c(k) e^{\theta} & 1
\end{array}\right)\left[\hat{Z}_{p}^{-}(k)\left(\begin{array}{cc}
1 & 0 \\
-c(k) e^{\theta} & 1
\end{array}\right)\right]^{-1}
$$

which shows that $\hat{R}(k)$ has actually no singularities at $k_{j}$. Similar considerations for $k \epsilon I I I$ show that $\hat{R}(k)$ has no singularities at $\bar{k}_{j}$ either. These facts together with $(1.19 \mathrm{~b})$ imply that $\hat{R}=I$.

The essence of deriving the statement (iii) is the usual idea of using Darboux transformations to solve an $\mathrm{RH}$ problem with signularities. We need to show that the function $\hat{Z}_{p}$ defined through (2.4.2)-(2.4.4) satisfies the RH problem (1.19), (2.4.1). In fact, by construction, the matrix $\hat{Z}_{p}$ satisfies the correct jump condition, and $\hat{Z}_{p} \rightarrow I$ as $k \rightarrow \infty$. Also,

where

$$
\begin{aligned}
& \begin{array}{l}
r e s \\
k_{j}
\end{array} \hat{Z}_{p}^{(1)}(x, t, k)=P_{j}\left(k_{j}\right)\left(k_{j} I+B_{j}\right) \hat{Z}_{j-1}\left(x, t, k_{j}\right)\left(\begin{array}{l}
1 \\
0
\end{array}\right) \frac{1}{\prod_{l=1, l \neq j}^{N}\left(k_{j}-k_{l}\right)} \\
& \qquad \hat{Z}_{p}^{(2)}\left(x, t, k_{j}\right)=P_{j}\left(k_{j}\right)\left(k_{j} I+B_{j}\right) \hat{Z}_{j-1}\left(x, t, k_{j}\right)\left(\begin{array}{c}
0 \\
1
\end{array}\right) \frac{1}{\Pi_{l=1}^{N}\left(k_{j}-\bar{k}_{l}\right)}
\end{aligned}
$$

$$
P_{j}(k)=\left(k I+B_{N}\right)\left(k I+B_{N-1}\right) \cdots\left(k I+B_{j+1}\right) .
$$

This yields equation (2.4.1a) as a direct consequence of equation (2.4.3a); actually, since det $P_{j}\left(k_{j}\right) \neq$ 0 these two equations are equivalent. Similarly, equation (2.4.1b) follows from (2.4.3b).

Due to the underline symmetry of the jump conditions, the regular $\mathrm{RH}$ problem for $Z(x, t, k)$ is always solvable (i.e. there exists vanishing Lemma [6]). In order to discuss the solvability of the algebraic equations (2.4.3), we rewrite them in the form

$$
B_{j}=-W_{j}\left(\begin{array}{cc}
k_{j} & 0 \\
0 & \bar{k}_{j}
\end{array}\right) W_{j}^{-1}, \quad W_{j} \doteqdot\left(\hat{Z}_{j-1}\left(x, t, k_{j}\right)\left(\begin{array}{c}
1 \\
-d_{j}(x, t)
\end{array}\right), \quad \hat{Z}_{j-1}\left(x, t, \bar{k}_{j}\right)\left(\begin{array}{c}
-\lambda \overline{d_{j}}(x, t) \\
1
\end{array}\right)\right) .
$$

This implies that the system of the algebraic equations for the matrices $B_{1}, \ldots, B_{N}$ is solvable iff

$$
\operatorname{det} W_{j} \neq 0, j=1, \ldots, N, \quad \forall x, t>0 \text {. }
$$

For $\lambda=-1$ (focusing case), equations (2.4.6) are always valid. Indeed, the symmetry

$$
\hat{Z}(x, t, k)=\sigma_{2} \overline{\hat{Z}(x, t, \bar{k})} \sigma_{2}
$$


yields the formula

$$
W_{1}=\sigma_{2} \bar{W}_{1} \sigma_{2}
$$

or

$$
\operatorname{det} W_{1}=\left|\left(W_{1}\right)_{11}\right|^{2}+\left|\left(W_{1}\right)_{21}\right|^{2}
$$

where $\left(W_{1}\right)_{11}$ and $\left(W_{1}\right)_{21}$ denote the 11 and 21 components of $W_{1}$. Since $\operatorname{det} \hat{Z}=1,\left(W_{1}\right)_{11}$ and $\left(W_{1}\right)_{21}$ cannot be zero simultaneously, and equation $(2.4 .6)$ is valid for $j=1$. The function $\hat{Z}_{1}$ satisfies the same symmetry condition $(2.4 .7)$ as $\hat{Z}$, and $\operatorname{det} \hat{Z}_{1}(x, t, k)=\left(k-k_{1}\right)\left(k-\bar{k}_{1}\right) \neq 0$ for $k=k_{2}, \bar{k}_{2}$; repeating the above arguments it follows that (2.4.6) is valid for $j=2$ together with the symmetry condition for $\hat{Z}_{2}$. Similar considerations apply to $j=3, \ldots, N$. This completes the proof of the Proposition 2.4.

In the defocusing case, $\lambda=1$, the above arguments regarding the solvability of the algebraic system (2.4.3) are not valid. In this case one has to replace $\sigma_{2}$ in equations (2.4.7) and (2.4.8) by $\sigma_{1}$. This implies that the + sign in $(2.4 .9)$ is replaced by the - sign. Actually, the asymptotic analysis of seciton 4 suggests that the solvability condition (2.4.6) does not hold in the defocusing case. In other words, solitons do not exist for $\lambda=1$.

\section{Rigorous Considerations}

We first discuss the $x$-problem. Let $\Phi(x, t, k)$ be defined by the integral equation (2.1.3). Let $\Psi(x, t, k)$ be defined by a similar integral equation with $\int_{0}^{x}$ replaced by $\int_{\infty}^{x}$. If $q(x, t) \epsilon L_{1}\left(\mathbb{R}^{+}\right)$in $x$, these integral equations have continuous and bounded solutions for fixed $t$ and $k$. Furthermore, $\Phi$ and $\Psi$ have the analytic dependence in $k$ indicated in equation (2.1.4). Also the RiemannLebesgue lemma implies that $\Phi, \Psi \rightarrow I$ as $k \rightarrow \infty$. If $q(x, t), x q(x, t) \epsilon L_{1}\left(\mathbb{R}^{+}\right) \cap L_{2}\left(\mathbb{R}^{+}\right)$in $x$, then $\psi(0, t, k) \epsilon H_{1}(\mathbb{R})$ in $k$.

In summary, if

$$
q(x, t), \quad x q(x, t) \epsilon L_{1}\left(\mathbb{R}^{+}\right) \cap L_{2}\left(\mathbb{R}^{+}\right) \text {in } x,
$$

then the formal results of $\S 2.1$ are justified. In particular the jump condition (1.13) is valid and the coefficients $\psi(0, t, k)-I$ of the jump matrix (2.1.6) belong to $H_{1}(\mathbb{R})$. 
We next consider the $t$-problem. Let $\hat{\Phi}(t, k)$ be defined by the integral equation (2.2.4). Let $\hat{\Psi}(t, k)$ be defined by a similar integral equation with $\int_{0}^{t}$ replaced by $\int_{\infty}^{t}$. If $q(0, t), q_{x}(0, t) \epsilon L_{1}\left(\mathbb{R}^{+}\right)$, these integral equations have continuous and bounded solutions for fixed $k$. Also $\hat{\Phi}$ and $\hat{\Psi}$ have the analytic dependence in $k$ indicated in equation (2.2.4), and $\hat{\Phi}, \hat{\Psi} \rightarrow I$ as $k \rightarrow \infty$. Furthermore, if in addition, $q(0, t) \epsilon L_{2}\left(\mathbb{R}^{+}\right)$and the first derivatives of $q(0, t)$ and $q_{x}(0, t) \epsilon L_{1}\left(\mathbb{R}^{+}\right)$, then $\hat{\Psi}(0, k)-I \epsilon L_{2}$ for $k^{2} \in \mathbb{R}$. Finally if in addition $t q(0, t), t q_{t}(0, t), t q_{t t}(0, t) \epsilon L_{\mathbf{1}}\left(\mathbb{R}^{+}\right)$, then $\hat{\Psi}(0, k)-I \epsilon H_{1}$ for $k^{2} \in \mathbb{R}$.

In summary, let

$$
v(t) \doteqdot q(0, t), \quad w(t) \doteqdot q_{x}(0, t)
$$

If

$$
v(t) \epsilon L_{1} \cap L_{2}\left(\mathbb{R}^{+}\right) ; \quad v^{\prime}(t), \quad w(t), \quad w^{\prime}(t), \quad t v(t), \quad t v^{\prime}(t), \quad t v^{\prime \prime}(t) \epsilon L_{1}\left(\mathbb{R}^{+}\right),
$$

then $\hat{\Psi}(t, k)$ and $\hat{\Phi}(t, k)$ exist and are related through a matrix $\hat{\Psi}(0, k)$ such that $\hat{\Psi}(0, k)-I \epsilon H_{1}$ for $k^{2} \in \mathbb{R}$.

In $\S 2 . \dot{2}$, we have formulated a $\mathrm{RH}$ problem which involves $\psi(0, t, k)$ in addition to $\hat{\Phi}(t, k)$ and $\hat{\Psi}(t, k)$. The function $\psi(0, t, k)$ satisfies equation (2.2.1) which is uniquely determined in terms of $v(t)$ and $w(t)$. On the other hand $\psi(x, 0, k)$ satisfies equation (1.3a) which is uniquely defined in terms of $u(x)=q(x, 0)$. The equality of $\psi(0, t, k)$ and $\psi(x, 0, k)$ at $x=t=0$ is guaranteed iff

$$
u(0)=v(0) .
$$

The relationship between $\psi(0, t, k), \hat{\Phi}(t, k)$, and $\hat{\Psi}(t, k)$ involves the matrices $\psi(0,0, k)$ and $\hat{\Psi}(0, k)$, which under the assumptions (3.1)-(3.4) belong to $H_{1}$ and have the analytic dependence indicated by the appropriate superscripts.

\section{Theorem 3.1 Assume that:}

(i) Equations (3.1), (3.3), and (3.4) are valid.

(ii) All the zeros of $\psi_{2}^{+}(0, t, k)$, of $s_{2}^{+}(k)$ (defined by equation (1.15a)), and of $\rho(k)$ (defined by equation $(1.15 \mathrm{~b})$ ) are simple, they do not coincide with each other, they do not lie on $k^{2} \epsilon \mathbb{R}$, and there is at most a finite number of them. The function $\rho(k)$ has no zeros if $\lambda=1$.

Then, the $2 \times 2$ matrix $\hat{Z}_{p}(x, t, k)$ defined by equation (1.18) has unit determinant and satisfies the jump conditions (1.19). The jump matrices are defined in terms of $\psi(0,0, k)$ and $\hat{\Psi}(0, k)$ which satisfy $\psi(0,0, k)-I, \hat{\Psi}(0, k)-I \epsilon H_{1}$. The matrix $\hat{Z}_{p}(x, t, k)$ is a meromorphic function in the complex 
$k$-plane cut along $\operatorname{Im}\left(k^{2}\right)=0$. Its possible poles can occur only at the zeros of $\rho(k)$ for $k \epsilon I I$, and at the complex conjugate of these zeros. The matrix $\hat{Z}_{p}(x, t, k)$ can be obtained by solving the RH problem of Figure 1.1. This $\mathrm{RH}$ is always solvable. The solution $q(x, t)$ of the NLS can be obtained from $q(x, t)=2 i \lim _{k \rightarrow \infty}\left(k \hat{Z}_{p}(x, t, k)\right)_{12}, k \in I$.

Proof. It follows from Proposition 2.1, 2.2 and the following properties of the jump matrices: (a) They are $H_{1}$ functions; (b) their product equals unity which guarantees continuity at $k=0$; (c) satisfy certain symmetry conditions involving complex conjugation and transposition. These symmetry conditions imply that there exist a vanishing lemma, [6], i.e. the homogeneous RH problem has only the zero solution.

QED

The main technical difficulty associated with the scheme presented in this paper is to find conditions of $u(x)$ and $v(t)$ which guarantee the assumption (3.1) as well as the assumption that $w(t), w^{\prime}(t) \epsilon L_{1}\left(\mathbb{R}^{+}\right)$.

It was shown in [12] that if

$$
u(x) \epsilon H_{2}\left(\mathbb{R}^{+}\right), \quad v \epsilon C_{2}\left(\mathbb{R}^{+}\right), \text {and } u(0)=v(0)
$$

then

$$
t \rightarrow q(, t) \text { is continuous from } \mathbb{R}^{+} \text {into } H_{2}\left(\mathbb{R}^{+}\right)
$$

and

$$
t \rightarrow q_{t}(, t) \text { is continuous from } \mathbb{R}^{+} \text {into } L_{2}\left(\mathbb{R}^{+}\right)
$$

Following Sung [13], we shall show that under some additional conditions on $u(x)$, the assumption (3.1) is valid.

Lemma 3.1 Assume that in addition to assumptions (3.5),

$$
x u(x) \text { and } x^{2} u(x) \epsilon L_{2}\left(\mathbb{R}^{+}\right)
$$

Then equation (3.1) is valid.

Proof. We shall first show that the assumption $x u(x) \epsilon L_{2}\left(\mathbb{R}^{+}\right)$implies that $q(x, t) \epsilon L_{1}\left(\mathbb{R}^{+}\right)$for fixed $t$.

The NLS equation and its complex conjugate imply 


$$
|q|_{t}^{2}=i\left(q_{x} \bar{q}-\bar{q}_{x} q\right)_{x}
$$

Thus

$$
\begin{aligned}
\partial_{t} \int_{0}^{\infty} x^{2}|q|^{2} \epsilon^{-\varepsilon x} d x & =i \int_{0}^{\infty} x^{2} e^{-\varepsilon x}\left(q_{x} \bar{q}-\bar{q}_{x} q\right)_{x} d x=-i \int_{0}^{\infty}\left(2 x-\varepsilon x^{2}\right) \epsilon^{-\varepsilon x}\left(q_{x} \bar{q}-\bar{q}_{x} q\right) d x \\
& \leq \int_{0}^{\infty}\left[4 x \left|q\left\|\left|q_{x}\right| e^{-\varepsilon x}+2 \varepsilon x^{2}\left|q \| q_{x}\right| e^{-\varepsilon x}\right] d x\right.\right. \\
& =4 \int_{0}^{\infty}\left|e^{-\frac{\varepsilon x}{2}} q_{x}\right|\left|x q e^{-\frac{\varepsilon x}{2}}\right| d x+4 \int_{0}^{\infty}\left|\frac{\varepsilon x}{2} e^{-\frac{\varepsilon x}{2}} q_{x}\right|\left|x q e^{-\frac{\varepsilon x}{2}}\right| d x
\end{aligned}
$$

The maximum of $\exp (-\varepsilon x / 2)$ and $\varepsilon x / 2 \exp (-\varepsilon x / 2)$ is 1 and $1 / e$ respectively, thus the l.h.s. of the above equation is not greater than

$$
4\left(1+\frac{1}{e}\right) \int_{0}^{\infty}\left|q_{x}\right|\left|x q e^{-\frac{\varepsilon x}{2}}\right| d x
$$

Let

$$
\varphi(t) \doteqdot\left\|x q(x, t) e^{-\frac{6 x}{2}}\right\|_{L_{2}^{x}\left(\mathbb{R}^{+}\right)},
$$

where $L_{2}^{x}\left(\mathbb{R}^{+}\right)$indicates $L_{2}$ in the variable $x$. Then

$$
\partial_{t}\left(\varphi^{2}\right) \leq 4\left(1+\frac{1}{e}\right) \max _{t \in[0, \infty)}\left\|q_{x}\right\|_{L_{2}^{x}\left(\mathbb{R}^{+}\right) \varphi}
$$

Assuming that $x q(x, 0) \epsilon L_{2}\left(\mathbb{R}^{+}\right)$and letting $\varepsilon \rightarrow 0$, equation $(3.9)$ yields that $x q(x, t) \epsilon L_{2}^{x}\left(\mathbb{R}^{+}\right)$.

Since $q(x, t)$ and $x q(x, t) \epsilon L_{2}^{x}\left(\mathbb{R}^{+}\right)$, the identity

implies that $q(x, t) \epsilon L_{1}^{x}\left(\mathbb{R}^{+}\right)$.

$$
\int_{0}^{\infty} q(x, t) d x=\int_{0}^{\infty}[(1+x) q] \frac{1}{(1+x)} d x
$$

We shall now show that the assumption $x^{2} u(x) \epsilon L_{2}\left(\mathbb{R}^{+}\right)$implies that $x^{2} q(x, t) \epsilon L_{2}^{x}\left(\mathbb{R}^{+}\right)$. Let

$$
\psi(t) \doteqdot\left\|x^{2} q e^{-\frac{\epsilon x}{2}}\right\|_{L_{2}^{x}\left(\mathbf{R}^{+}\right)} .
$$

Similar considerations as above yield

$$
\partial_{t} \psi^{2} \leq\left(8+\frac{4}{\epsilon}\right) \int_{0}^{\infty}\left|x q_{x}\right|\left|x^{2} q e^{-\frac{\kappa x}{2}}\right| d x \leq\left(8+\frac{4}{e}\right)\left\|x q_{x}\right\|_{L_{2}^{x}\left(\mathbb{R}^{+}\right)} \psi .
$$

Thus if $x^{2} q(x, 0) \epsilon L_{2}\left(\mathbb{R}^{+}\right), \psi$ exists, and since $x^{2} q(x, t) \epsilon L_{2}^{x}\left(\mathbb{R}^{+}\right)$it follows that $x q(x, t) \epsilon L_{1}^{x}\left(\mathbb{R}^{+}\right)$. QED 


\section{The Asymptotic Analysis}

In order to determine the large $t$ behavior of the solution $q(x, t)$ one needs to study the large $t$ asymptotic behavior of the oscillatory RH problem (1.19). The corresponding problem for integrable equations on the full line was first studied in [7] (see also [8], [11] and the review [9]). A rigorous and elegant approach to studying the asymptotic behavior of $\mathrm{RH}$ problems has been recently developed in [10]. In what follows we shall use this new approach.

We first study the solution $\hat{Z}(x, t, k)$ of the regular $\mathrm{RH}$ problem corresponding to the $\mathrm{RH}$ problem (1.19) (see Proposition 2.4 (iii)).

Theorem 4.1 Under the assumptions of Proposition 2.4, the solution $\hat{Z}(x, t, k)$ of the regular $\mathrm{RH}$ problem corresponding to (1.19) satisfies the asymptotic equation,

$$
\hat{Z}(x, t, k)=\left(I+O\left(t^{-\frac{1}{2}}\right)\right)(\delta(k))^{\sigma_{3}}, \quad t \rightarrow \infty, \quad 0<A \leq \frac{x}{t} \leq B<\infty,
$$

uniformly for $|\operatorname{Imk}| \geq \varepsilon>0$. The scalar function $\delta(k)$ is given by

$$
\delta(k)=\exp \left[\frac{1}{2 \pi i} \int_{-\infty}^{k_{0}} \frac{\ln \left(1-\lambda\left|b\left(k^{\prime}\right)-\lambda \overline{c\left(k^{\prime}\right)}\right|^{2}\right)}{k^{\prime}-k} d k^{\prime}\right], \quad k_{0}=-\frac{x}{4 t}
$$

Proof The method of [10] can be thought of as a nonlinear steepest descent method. The stationary point, and the directions of the steepest descent associated with exp $2 i\left(k x+2 k^{2} t\right)$, are given by $k_{0}=-x / 4 t$, and by $\operatorname{Im}\left(i\left(k-k_{0}\right)^{2}\right)=0$, respectively. This implies that we must deform the original RH problem to one defined on the above steepest descent contours (see the solid lines of Figure 4.1). We now discuss how this deformation can be achieved. 


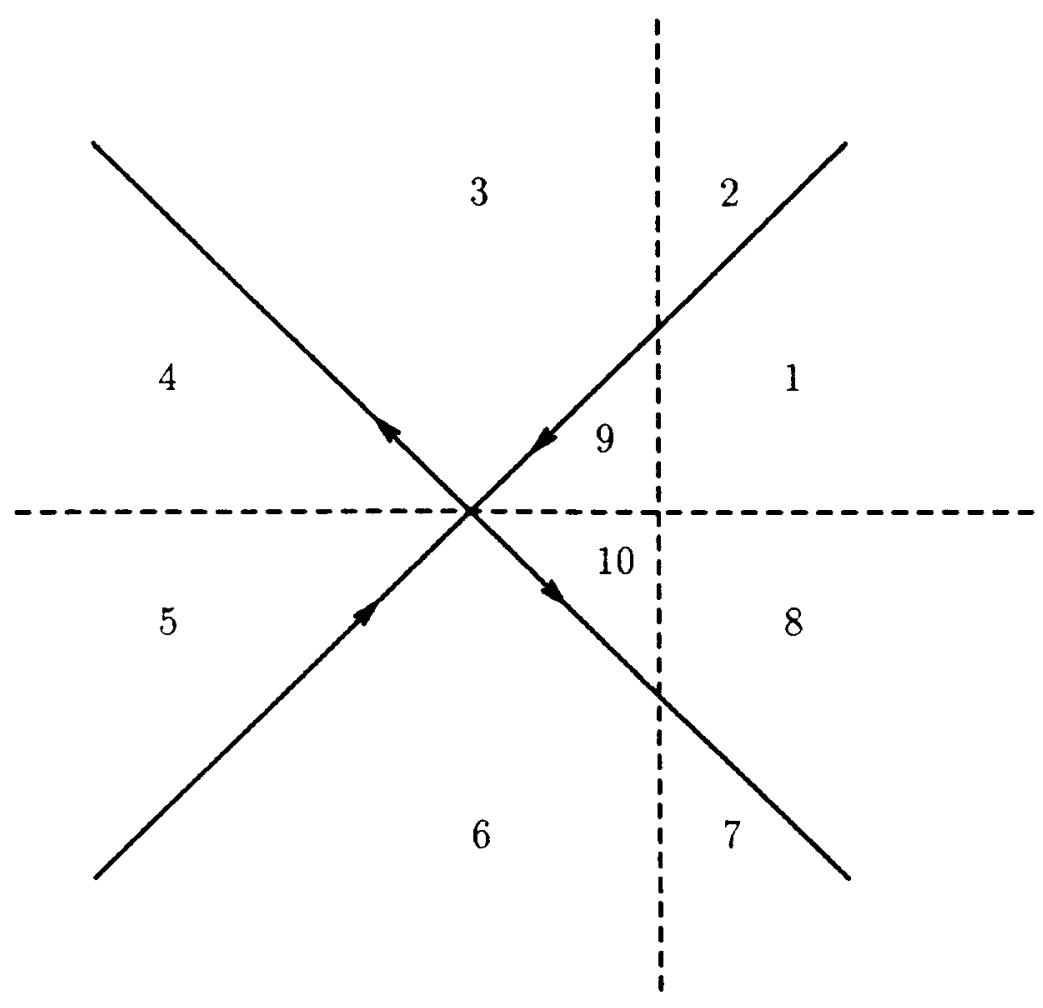

Figure 4.1

The jump matrix along the positive real axis can be factorized into two triangular matrices. Using this factorization we find

$$
\hat{Z}^{-}\left(\begin{array}{cc}
1 & b_{0}(k) e^{-\theta} \\
0 & 1
\end{array}\right)=\hat{Z}^{+}\left(\begin{array}{cc}
1 & 0 \\
\lambda \overline{b_{0}(k)} e^{\theta} & 1
\end{array}\right), k \in \mathbb{R}^{+} .
$$

Suppose that $b_{0}(k)$ is a rational function with appropriately chosen poles such that $b_{0}(k)$ is analytic in region 8 . Since $e^{-\theta}$ and $e^{\theta}$ are analytic and decreasing in regions 8 and 1 respectively, it follows that the relevant jump matrices can be absorbed into $\hat{Z}^{-}$and $\hat{Z}^{+}$. In this way one can eliminate the jump along $\mathbb{R}^{+}$. Let $c_{0}$ and

$$
r_{0}(k) \doteqdot b_{0}(k)-\lambda \overline{c_{0}(k)}
$$

be appropriate rational functions. Then the regions 9 and 10 can be handled without difficulty since the factorization of the jump matrix for $k_{0}<k<0$ still has the "correct" triangularity. Also, the jumps along the broken lines separating regions 1 and 9 and regions 8 and 10 disappear. However, the factorization of the jump matrix for $k<k_{0}$ has the "wrong" triangularity, 


$$
\hat{Z}^{-}=\hat{Z}^{+}\left(\begin{array}{cc}
1 & r_{0} e^{-\theta} \\
0 & 1
\end{array}\right)\left(\begin{array}{cc}
1 & 0 \\
-\lambda \bar{r}_{0} e^{\theta} & 1
\end{array}\right),
$$

i.e. the jump matrix involving $e^{-\theta}$ cannot be absorbed into $\hat{Z}^{+}$. To overcome this problem, one introduces the function $\delta(k)$. This function is analytic in the complex $k$-plane cut along $\left(-\infty, k_{0}\right]$; along this cut it satisfies the jump condition

$$
\delta_{+}(k)=\delta_{-}(k)\left(1-\lambda|b(k)-\lambda \bar{c}(k)|^{2}\right), \quad k \epsilon\left(-\infty, k_{0}\right] .
$$

This jump is precisely chosen by the requirement of reversing the triangularity of the jump along $\left(-\infty, k_{0}\right)$. Indeed, if

$$
\hat{W}(x, t, k)=\hat{Z}(x, t, k)(\delta(k))^{-\sigma_{3}},
$$

and if $G$ denotes the jump matrices associated with $\hat{Z}$, then the jump matrices associated with $\hat{W}$ are given by $G_{0}=\delta^{\sigma_{3}} G \delta^{-\sigma_{3}}$. The jump matrices of the RH problem

$$
\hat{W}^{-}(x, t, k)=\hat{W}^{+}(x, t, k) G_{0}(x, t, k), \quad k^{2} \in \mathbb{R} ; \hat{W} \rightarrow I, \quad k \rightarrow \infty,
$$

posses the correct triangular factorizations:

$$
\begin{gathered}
G_{0}(x, t, k)=\left(\begin{array}{ccc}
1 & 0 \\
\lambda \bar{b}_{0}(k) \delta^{-2}(k) e^{\theta} & 1
\end{array}\right)\left(\begin{array}{cc}
1 & -b_{0}(k) \delta^{2}(k) e^{-\theta} \\
0 & 1
\end{array}\right), k \in \mathbb{R}^{+}, \\
G_{0}(x, t, k)=\left(\begin{array}{ccc}
1 & r_{0}(k) \delta^{2}(k) e^{-\theta} \\
0 & 1
\end{array}\right)\left(\begin{array}{cc}
1 & 0 \\
-\lambda \bar{r}_{0}(k) \delta^{-2} e^{\theta} & 1
\end{array}\right), \quad k \epsilon\left[k_{0}, 0\right], \\
G_{0}(x, t, k)=\left(\begin{array}{cc}
1 & 0 \\
-\frac{-\lambda r_{0}(k) \delta^{-2}(k)}{1-\lambda\left|r_{0}(k)\right|^{2}} e^{\theta} & 1
\end{array}\right)\left(\begin{array}{cc}
1 & \frac{r_{0}(k)}{1-\lambda\left|r_{0}(k)\right|^{2}} \delta_{+}^{2}(k) e^{-\theta} \\
0 & 1
\end{array}\right), k<k_{0},
\end{gathered}
$$

where $\delta_{ \pm}(k)=\delta(k \pm i 0), k \in \mathbb{R}$. The first two equations above follow from equations $(4.3)$ and (4.4). For the derivation of the third equation above we use equation (4.6) and the fact that $|b(k)-\lambda \bar{c}(k)|=\left|r_{0}\right|$ (see the definitions of $b_{0}$ and $c_{0}$ in proposition 2.4 (iii)).

The triangular factorizations of $G_{0}$ imply that the jumps along the real axis can be eliminated. The jump condition along the broken line separating regions 2 and 3 is given by 


$$
\hat{W}^{-}=\hat{W}^{+}\left(\begin{array}{cc}
1 & 0 \\
c_{0} \delta^{-2} e^{\theta} & 1
\end{array}\right)
$$

this shows that the jump matrix can be absorbed into $\hat{W}^{+}$. Similarly for the jump along the broken line separating regions 6 and 7 . Hence, the function,

$$
X(x, t, k)=\hat{W}(x, t, k) K(x, t, k)
$$

where $K(x, t, k)$ is given by

$$
\begin{gathered}
\left(\begin{array}{cc}
1 & 0 \\
\lambda \overline{b_{0}(\bar{k})} \delta^{-2}(k) e^{\theta} & 1
\end{array}\right),\left(\begin{array}{cc}
1 & 0 \\
c_{0}(k) \delta^{-2}(k) e^{\theta} & 1
\end{array}\right), I,\left(\begin{array}{cc}
1 & -\frac{r_{0}(k)}{1-\lambda r_{0}(k) r_{0}(\bar{k})} \delta^{2}(k) e^{-\theta} \\
0 & 1
\end{array}\right), \\
\left(\begin{array}{cc}
1 & 0 \\
-\lambda \frac{\overline{r_{0}(\bar{k})}}{1-\lambda r_{0}(k) r_{0}(\bar{k})} \delta^{-2}(k) e^{\theta} & 1
\end{array}\right), I,\left(\begin{array}{cc}
1 & \lambda \overline{c_{0}(\bar{k})} \delta^{2}(k) e^{-\theta} \\
0 & 1
\end{array}\right), \\
\left(\begin{array}{cc}
1 & b_{0}(k) \delta^{2}(k) e^{-\theta} \\
0 & 1
\end{array}\right),\left(\begin{array}{cc}
1 & 0 \\
\lambda \overline{r_{0}(\bar{k})} \delta^{-2}(k) e^{\theta} & 1
\end{array}\right),\left(\begin{array}{cc}
1 & r_{0}(k) \delta^{2}(k) e^{-\theta} \\
0 & 1
\end{array}\right),
\end{gathered}
$$

for $k \epsilon 1,2, \ldots, 9,10$ respectively is a sectionally holomorphic function satisfying the $\mathrm{RH}$ problem depicted in Figure 4.2 (also $X \rightarrow I$ as $k \rightarrow \infty$ ).

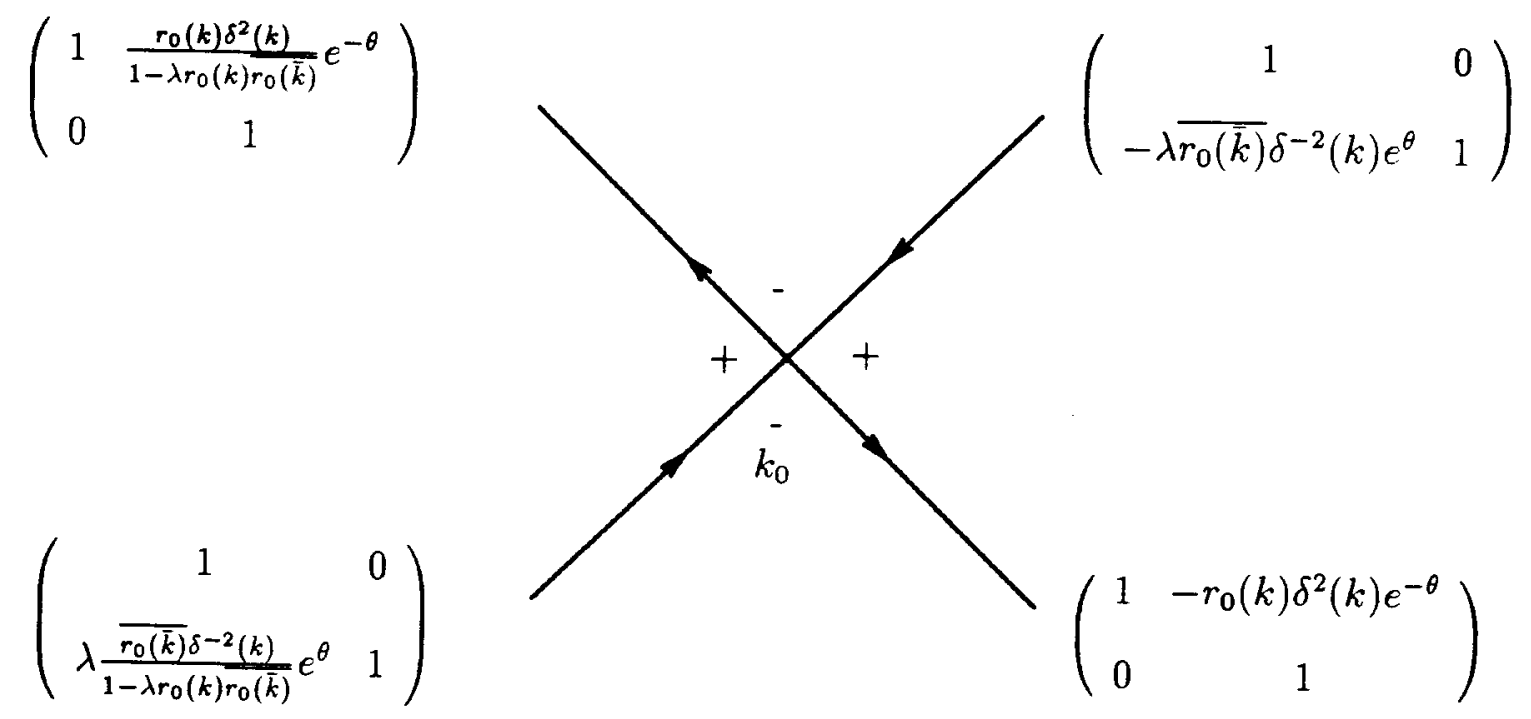

Figure 4.2 
The deformed RH problem arising in the asymptotic analysis of the initial-boundary value problem of NLS. The $x, t$ dependence enters only through $\theta(x, t)=2 i\left(k x+2 k^{2} t\right)$ and $\delta=\delta\left(k ; k_{0}\right), k_{0}=-x / 4 t$.

The $\mathrm{RH}$ for $X(x, t, k)$ has the crucial property that its jump matrices decay exponentially to the identity away from the stationary point $k_{0}$. This, just like the classical steepest descent method, implies

$$
X(x, t, k)=I+O\left(t^{-\frac{1}{2}}\right)
$$

for $k$ away from $k_{0}$. Indeed, if $\Gamma$ and $\tilde{G}_{0}$ denote the contours and the jump matrices of Figure 4.2, then

$$
X(x, t, k)=I+\frac{1}{2 \pi i} \int_{\Gamma} \frac{X^{+}\left(x, t, k^{\prime}\right)\left(I-\tilde{G}_{0}\left(x, t, k^{\prime}\right)\right.}{k^{\prime}-k} d k^{\prime},
$$

for $k$ not on $\Gamma$. Taking into account the apriori estimate $\left|X^{+}(x, t, k)\right| \leq$ const, and using the classical Laplace's method to the integral of the r.h.s. of equation (4.11), one finds equation (4.10).

This concludes the proof of Theorem 4.1 in the case that $b_{0}$ and $c_{0}$ are rational. The general case can be reduced to this case following the construction of [10].

Remark 4.1 In the solitonless case $\hat{Z}_{p}=\hat{Z}$, and Theorem 4.1 gives the asymptotic solution of the $\mathrm{RH}$ problem (1.19). Using equation (2.3.1b) which relates $q(x, t)$ to $\hat{Z}$, and the asymptotic relation (4.1), it follows that in the solitonless case

$$
q(x, t)=O\left(t^{-\frac{1}{2}}\right), \quad t \rightarrow \infty, \quad 0 \leq A \leq \frac{x}{t} \leq B .
$$

We now consider the case that poles $\left\{k_{j}\right\}_{1}^{N}$ do exist. We assume $\lambda=-1$.

Theorem 4.2 Under the assumptions of Proposition 2.4, the solution $\hat{Z}_{p}(x, t, k)$ of the RH problem (1.19) satisfies the asymptotic equation

$$
\hat{Z}_{p}(x, t, k)=\left(I+O\left(t^{-\frac{1}{2}}\right)\right) \hat{Z}_{S}(x, t, k)(\delta(k))^{\sigma_{3}}, \quad t \rightarrow \infty, \quad 0<A \leq \frac{x}{t} \leq B<\infty
$$


uniformly for $|\operatorname{Im} k| \geq \varepsilon>0$. The scalar funciton $\delta(k)$ is defined in equation (4.2). The matrix funciton $\hat{Z}_{S}(x, t, k)$ is given by the algebraic equations $(2.4 .2)-(2.4 .4)$ with $\lambda=-1$, with $\hat{Z}$ replaced by $I$, and with $c_{j}$ replaced by $c_{j}\left(\delta\left(k_{j}\right)\right)^{-2}$.

Proof Equation (4.13) is a direct consequence of Proposition 2.4, of Theorem 4.1, and of the equation $\overline{\delta\left(k_{j}\right)}=\left(\delta\left(\bar{k}_{j}\right)\right)^{-1}$.

Remark 4.2 The function $\hat{Z}_{S}$ is nothing but the matrix eigenfunction corresponding to the pure $N$-soliton solution $q_{s}(x, t)$ of the NLS equation with parameters $\left\{k_{j}\right\}_{1}^{N}$ and $\left\{c_{j}\left(\delta\left(k_{j}\right)\right)^{-2}\right\}_{1}^{N}$. This implies that

$$
q(x, t)=q_{S}(x, t)+O\left(t^{-\frac{1}{2}}\right)
$$

Using the well-known formula for the $N$-soliton soution of the NLS (or the algebraic system (2.4.3) directly), one can easily extract from (4.14) the asymptotics of $q(x, t)$ along the soliton rays, given in (1.4) and (1.5).

Remark 4.3 Just like in the classical steepest descent method, one can improve the estimate (4.10) by calculuating the contribution from the stationary point $k_{0}$ in closed form. It turns out [11] that the corresponding model RH problem can be solved explicitly in terms of parabolic cylindrical functions. The analysis is similar to that for the full line problem presented in [9]. This leads to the following formula for the dispersive part of the asymptotics of $q(x, t)$ :

$$
q(x, t)=\alpha\left(-\frac{x}{4 t}\right) t^{-1 / 2} \exp \left\{\frac{i x^{2}}{4 t}+2 i \alpha^{2}\left(-\frac{x}{4 t}\right) \log t+i \varphi_{0}\left(-\frac{x}{4 t}\right)\right\}+0\left(t^{-1 / 2}\right),
$$

where the amplitude $\alpha$ and the phase $\varphi_{0}$ are given by

$$
\begin{gathered}
\alpha^{2}(k)=\frac{1}{4 \pi} \log \left[1+|b(k)+\overline{c(k)}|^{2}\right] \\
\varphi_{0}(k)=2 \alpha^{2}(k) \log 2+\frac{3 \pi}{4}+\arg (b(k)+\overline{c(k)}) \\
+\arg \Gamma\left(-2 i \alpha^{2}(k)\right)+4 \int_{-\infty}^{k} \log |\mu-k| d \alpha^{2}(\mu)+2 \sum_{j=1}^{N} \arg \left(k_{j}-k\right) \operatorname{sign}\left(\xi_{j}-k\right) .
\end{gathered}
$$


Remark 4.4 One can use equation (4.16a) to replace $\log \left[1+|b(k)+\overline{c(k)}|^{2}\right]$ in the integral terms of equations (1.5) by $4 \pi \alpha^{2}$. This shows that indeed these terms represent the interaction of solitons with the dispersive part.

Remark 4.5 In the case of the NLS on the full line one can use equations (4.16) to solve $b$ and $c$ in terms of $\alpha$ and $\varphi_{0}$. However, in our case this can not be done since to define $b$ and $c$ one needs two real functions defined on the whole linear (see Remark 2 in section 2) while $\alpha$ and $\varphi_{0}$ are real functions defined in the half-line. This is a reflection of the fact that the information traveling towards the boundary is lost as $t \rightarrow \infty$. In the case of zero initial data, waves travel away from the boundary, no information is lost asymptotically, and the asymptotics of $q(x, t)$ can be used to recover $c$ and hence $q(0, t)$.

\section{Acknowledgements}

This work was partially supported by the National Science Foundation under Grant Number DMS-9111611, and the Air Force Office of Scientific Research under Grant Number AFOSR-890510. The authors are thankful to L. Sung for many useful suggestions and to I.M. Gel'fand for his encouragement. The idea of using a combination of exact methods and of PDE techniques to solve initial-boundary value problems emerged after many discussions with $\mathrm{J}$. Bona.

\section{References}

[1] P. Lax, Comm. Pure Appl. Math., 21, 467 (1968).

[2] J. Sheerin et al, J. Atmos. Terr. Phys. 44, 1043 (1982).

[3] J.V. Moloney and A.C. Newell, Phys. Rev. A. 39, 1809-1840 (1989).

[4] A.S. Fokas, Proceedings of the III Potsdam - V Kiev International Workshop 1991, SpringerVerlag, Berlin, 1992. A.S. Fokas and A.R. Its, Phys. Rev. Lett., 68, 3117 (1992).

[5] V. Zakharov and A. Shabat, Soviet Phys. JETP, 34, 62 (1972).

[6] X. Zhou, SIAM J. Math. Anal. 20, 966-986 (1989).

[7] S.V. Manakov, Sov. Phys. - JETP, 38, No. 4, 693-696, (1974).

[8] M.J. Ablowitz and H. Segur, Stud. Appl. Math., 57 13-24 (1977). 
[9] P. Deift, A. Its, and X. Zhou, Long-time Asymptotics for Integrable Nonlinear Wave Equations, in Important Developments of Soliton Theory, ed. A.S. Fokas, V. Zakharov, Springer-Verlag. 1993.

[10] P. Deift and X. Zhou, Announcement in Bull. Amer. Math. Soc., (New Series), 26, 119-123 (1992).

[11] A.R. Its, Sov. Math. Dokl., 24, No. 3, 452-456 (1981).

[12] R. Caroll and C. Bu, Solution of the Forced NLS using PDE Techniques, Appl. Anal. 41, 33-51 (1991).

[13] L. Sung, Solution of the Initial-Boudnary Value Problem of NLS using PDE Techniques, preprint, Clarkson University, 1993.

[14] A.S. Fokas, Physica D, 35, 167-185 (1989).

[15] M.J. Ablowitz and H. Segur, J. Maht. Phys. 16, 1054 (1975).

[16] E.K. Sklyanin, Funk. Analiz. (Func. Anal. Appl.) 21 (2), 86-7 (1987).

[17] V.O. Tarasov, Zap. Nauch. Semin. LOMI 169, 151 (1988).

[18] R.F. Bikbaev and A.R. Its, Math. Notes 453 (1989) (Russian).

[19] I.T. Habibullin, Nonlinear and Turbulent Processes, vol. 1 (Singapore: World Scientific) 259 (1990).

[20] J. Bona, W.G. Pritchard, and L.R. Scott, Philos. Trans. R. Soc. London A 302, 457-510 (1981).

[21] J. Bona and R. Winther, SIAM J. Math. Anal. 14, 1056-1106 (1983).

[22] J. Bona and R. Winther, Differ. Integral Equations 2, 228-250 (1989).

[23] R.L. Chou and C.K. Chu, Phys. Fluids A 2, 1574 (1990); R.L. Chou, Ph.D. dissertation, City University of New York, 1987 (unpublished).

[24] C.K. Chu and R.L. Chou, Adv. Appl. Mech. 27, 283-302 (1990); D.J. Kaup and P. Wycoff, Stud. Appl. Math. 81, 7-20 (1989).

[25] E.S. Gutshabash, V.D. Lipovsky, Zap. Nauch. Semin. LOMI, 180, 53-62 (1990). 